\title{
Neanthes goodayi sp. nov. (Annelida, Nereididae), a remarkable new annelid species living inside deep-sea polymetallic nodules
}

\author{
Regan DRENNAN ${ }^{\oplus_{1, *}}$, Helena WIKLUND ${ }^{\oplus_{2}}$, Muriel RABONE ${ }^{\oplus_{3}}$, \\ Magdalena N. GEORGIEVA ${ }^{\oplus_{4}}$, Thomas G. DAHLGREN ${ }^{\oplus_{5}}$ \& Adrian G. GLOVER ${ }^{\odot_{6}}$ \\ ${ }^{1}$ Ocean \& Earth Science, University of Southampton, European Way, Southampton SO14 3ZH, UK. \\ 1,2,3,4,6 Life Sciences Department, Natural History Museum, London SW7 5BD, UK 2. \\ ${ }^{2,5}$ Department of Marine Sciences, University of Gothenburg, Box 463, 40530 Gothenburg, Sweden. \\ ${ }^{2,5}$ Gothenburg Global Biodiversity Centre, Box 463, 40530 Gothenburg, Sweden. \\ ${ }^{5}$ NORCE Norwegian Research Centre, Bergen, Norway. \\ *Corresponding author: r.drennan@nhm.ac.uk \\ ${ }^{2}$ Email: helena.wiklund@marine.gu.se \\ ${ }^{3}$ Email: M.Rabone@nhm.ac.uk \\ ${ }^{4}$ Email: m.georgieva@nhm.ac.uk \\ ${ }^{5}$ Email: thda@norceresearch.no \\ ${ }^{6}$ Email: a.glover@nhm.ac.uk
}

\footnotetext{
${ }^{1}$ urn:lsid:zoobank.org:author:63A79724-B7EC-4D7D-99BF-2B3CB2EBCD6D

${ }^{2}$ urn:lsid:zoobank.org:author:114C3853-7E48-42AC-88F3-F7AC327B24F3

${ }^{3}$ urn:1sid:zoobank.org:author:0D08F91D-A967-4A8B-A244-9963239F044A

${ }^{4}$ urn:1sid:zoobank.org:author:76E89A87-2986-4574-895E-2FFF0743CDD6

${ }^{5}$ urn:1sid:zoobank.org:author:B24FC764-1770-41EA-8CF8-E1CDD0C4E489

${ }^{6}$ urn:lsid:zoobank.org:author:03878173-9B00-462A-98BC-4E246E3D32FF
}

\begin{abstract}
A new species of abyssal Neanthes Kinberg, 1865, N. goodayi sp. nov., is described from the Clarion-Clipperton Zone in the central Pacific Ocean, a region targeted for seabed mineral exploration for polymetallic nodules. It is a relatively large animal found living inside polymetallic nodules and in xenophyophores (giant Foraminifera) growing on nodules, highlighting the importance of the mineral resource itself as a distinct microhabitat. Neanthes goodayi sp. nov. can be distinguished from its congeners primarily by its distinctive, enlarged anterior pair of eyes in addition to characters of the head, pharynx and parapodia. Widespread, abundant, and easily recognisable, $N$. goodayi sp. nov. is also considered to be a suitable candidate as a potential indicator taxon for future monitoring of the impacts of seabed mining.
\end{abstract}

Keywords. CCZ, molecular phylogeny, Polychaeta, morphology, abyssal fauna.

Drennan R., Wiklund H., Rabone M., Georgieva M.N., Dahlgren T.G. \& Glover A.G. 2021. Neanthes goodayi sp. nov. (Annelida, Nereididae), a remarkable new annelid species living inside deep-sea polymetallic nodules. European Journal of Taxonomy 760: 160-185. https://doi.org/10.5852/ejt.2021.760.1447 


\section{Introduction}

Exploration of our deep oceans for potential new industrial activities has increased rapidly in recent decades with the so-called 'blue growth' economy (European Commission 2020). Critical to a sustainable blue economy is baseline knowledge on the environmental characteristics of these exploration areas, in particular knowledge of the species that live there (Glover et al. 2018). This is especially the case in the central Pacific abyss Clarion-Clipperton Zone (CCZ), a region targeted for seabed mineral exploration for polymetallic nodules, where basic faunistic and taxonomic data are notably lacking and many animals likely undescribed or undocumented (Glover et al. 2018). Here, we describe a new nereidid annelid from the abyss that is not only important for understanding the general baseline biology of the region, but also presents a remarkable natural history - living inside the polymetallic nodules themselves. As the species is relatively large and easy to recognise, it should be added to a list of nodule-dwelling fauna that could be used as indicators in future environmental assessments (Lim et al. 2017). Information on the existence, abundance and distribution of these species could be essential to environmental monitoring and conservation measures in the region.

The CCZ lies in international waters and lacks strictly defined boundaries; however, it is generally accepted to encompass the region between the Clarion and Clipperton Fracture Zones, with multiple polymetallic nodule exploration contracts for seabed minerals issued by the International Seabed Authority (ISA 2018), extending from $115^{\circ} \mathrm{W}$ (the easternmost extent of the UK-1 polymetallic nodule exploration area) to approximately $158^{\circ} \mathrm{W}$ (the westernmost extent of the COMRA polymetallic nodule exploration area). As such, we hereafter use a working definition of the $\mathrm{CCZ}$ as comprising the box: $13^{\circ} \mathrm{N}, 158^{\circ} \mathrm{W} ; 18^{\circ} \mathrm{N}, 118^{\circ} \mathrm{W} ; 10^{\circ} \mathrm{N}, 112^{\circ} \mathrm{W} ; 2^{\circ} \mathrm{N}, 155^{\circ} \mathrm{W}-$ an area spanning almost 6 million $\mathrm{km}^{2}$, approximately $1.4 \%$ of the ocean's surface.

Polymetallic nodules are small mineral accretions (usually $5-10 \mathrm{~cm}$ in diameter, but occasionally exceeding $20 \mathrm{~cm}$ ) rich in cobalt, manganese, copper and nickel, among numerous other metals of economic interest (Hein et al. 2013). These nodules sit on the sea floor, often half submerged in sediment, providing the only hard substrate in an otherwise soft sediment environment, contributing to a high habitat heterogeneity compared with regions of the deep sea without nodules or hard substrate. Nodules provide microhabitats for meio- and macrofaunal groups such as annelids and crustaceans (Thiel et al. 1993; Gooday et al. 2017; Kersken et al. 2019), in addition to sites of attachment for sessile megafauna (e.g., Relicanthus sp. anemones) (Amon et al. 2016).

Nereididae de Blainville, 1818 is among the most diverse families within Annelida, with over 40 valid genera and up to 750 valid species (Read \& Fauchald 2020a). Members of the family are broadly omnivorous, and most species appear to be facultatively motile, rarely leaving mucus-built tubes and burrows unless disturbed or when conditions become unfavourable (Fauchald \& Jumars 1979; Jumars et al. 2015). Sexually mature individuals may develop into pelagic morphs (epitokes), which are thought to have much greater motility. However, not all nereidids form epitokes during reproduction, and not all epitokes are pelagic, with the degree of modification varying between species and sexes (Bakken \& Wilson 2005).

The genus Neanthes Kinberg, 1865 is one of the most diverse genera within the family, with over 80 currently accepted species (Read \& Fauchald 2020b), and can be distinguished from similar genera such as Hediste Malmgren, 1867 and Nereis Linnaeus, 1758 by morphological characters primarily relating to the presence or absence of certain chaetal types, for example in lacking compound falcigers in notopodial fascicles (as in Nereis), but possessing homogomph spinigers in ventral neuropodial fascicles (absent in Hediste and Nereis) (Bakken \& Wilson 2005). 
However, Neanthes is considered to be polyphyletic (Bakken \& Wilson 2005) and a generic revision based on phylogenetic analyses is needed to resolve its taxonomy (Bakken \& Wilson 2005; Bakken 2006; Glasby et al. 2011; Shimabukuro et al. 2017; Villalobos-Guerrero 2019). The majority of species of Neanthes have been described from shallow or intertidal waters, with only 13 species reported from depths greater than $200 \mathrm{~m}$ (Khlebovich 1996; Shimabukuro et al. 2017; Hsueh 2019). Notably, Thiel et al. (1993), when examining nodules collected from the South Pacific (outside of the CCZ) as part of the DISCOL project, reported two unidentified species of Neanthes when first describing polymetallic nodule crevices as a discrete microhabitat; these were among six annelid taxa that were only found within interstitial mud from nodule crevices, and not from the surrounding soft sediment.

In this study, we describe a new species of abyssal Neanthes observed to reside either directly within nodule crevices, within mud balls on nodule surfaces or burrowing within xenophyophores (giant foraminiferans) growing on nodules. This species is notable in that it highlights the potential importance of nodule microhabitats for macrofaunal-sized animals, and is also one of the most abundant and widespread annelid species collected as part of the ABYSSLINE ('ABYSSal baseLINE') UK-1 environmental survey project. Easily recognisable, it is a critical 'target taxon' for further assessments of biogeography and population connectivity patterns, the subject of a separate study (Dahlgren et al., unpublished data).

\section{Material and methods}

\section{Fieldwork}

Specimens were collected across two cruises, the first UK Seabed Resources ABYSSLINE cruise (AB01) sampling the UK-1 exploration contract area aboard the RV Melville, October 2013, and the second cruise (AB02) sampling the UK-1 and OMS (Ocean Mineral Singapore) exploration contract areas as well as an area to the north designated as Area of Particular Environmental Interest 6 (APEI-6) onboard RV Thomas G. Thompson, February-March 2015 (Fig. 1). A comprehensive description of the DNA taxonomy methodological pipeline used here is provided in Glover et al. (2016). In summary, a range of oceanographic sampling gear, including box corer, epibenthic sledge (EBS), ROV and multiple corer, were used to collect deep-sea benthic specimens from the UK-1, OMS and APEI-6 areas. Geographic data from sampling activities were recorded on a central GIS database. A 'cold-chain' pipeline was used in the live-sorting of specimen samples aboard both vessels, where material was constantly maintained in chilled, filtered seawater held at $2-4{ }^{\circ} \mathrm{C}$. Specimens underwent preliminary identification at sea and were live-imaged using digital cameras attached to stereo microscopes (Glover et al. 2016). All specimens were then stored in individual microtube vials containing an aqueous solution of $80 \%$ nondenatured pre-chilled ethanol, which were numbered, barcoded into a database and stored chilled until return to the Natural History Museum, London, UK.

\section{Laboratory work}

A total of 43 specimens were identified as conspecific using genetic data (see below) and considered in morphological analyses, with a portion of representative specimens selected as type material for more detailed analyses.

Specimen measurements taken included total length (TL), length to chaetiger 15 (L15), width of chaetiger 15 excluding parapodia (W15), and the total number of chaetigers for complete specimens. Paragnaths for each pharangeal area were counted, with paired areas that differed in numbers distinguished using $a$ and $b$ for the left and right side of the specimen respectively. The number of teeth on the jaws were also counted. For specimens where the pharynx was not everted, a longitudinal dissection was made in the mid-ventral region. For examination of parapodial features and modifications along the body, several 
parapodia were removed (from chaetigers $1,3,6,10$, every tenth chaetiger thereafter, and a posteriormost chaetiger, where possible) and mounted on glass slides. Parapodia were dissected from either the left or right side of the specimen based on intactness of features such as cirri.

Specimens were examined using compound and light microscopes, and photographed using attached digital cameras on both microscopes. Figures were assembled using Adobe Photoshop CS6 software. A fine white or black line was used to outline and highlight particular morphological features where they were unclear from the images alone. Standardised terminology of nereidid parapodial features follows Villalobos-Guerrero \& Bakken (2018); the shape of pharangeal areas and ridge patterns follows Villalobos-Guerrero (2019).

A small tissue sample was taken from each specimen for DNA extraction. The DNeasy Blood and Tissue Kit (Qiagen) was used to extract DNA using a Hamilton Microlab STAR Robotic Workstation. Approximately 1800 bp of 18 S rRNA(18S) were amplified using the primers 18SA 5'-AYCTGGTTGATCCTGCCAGT-3'
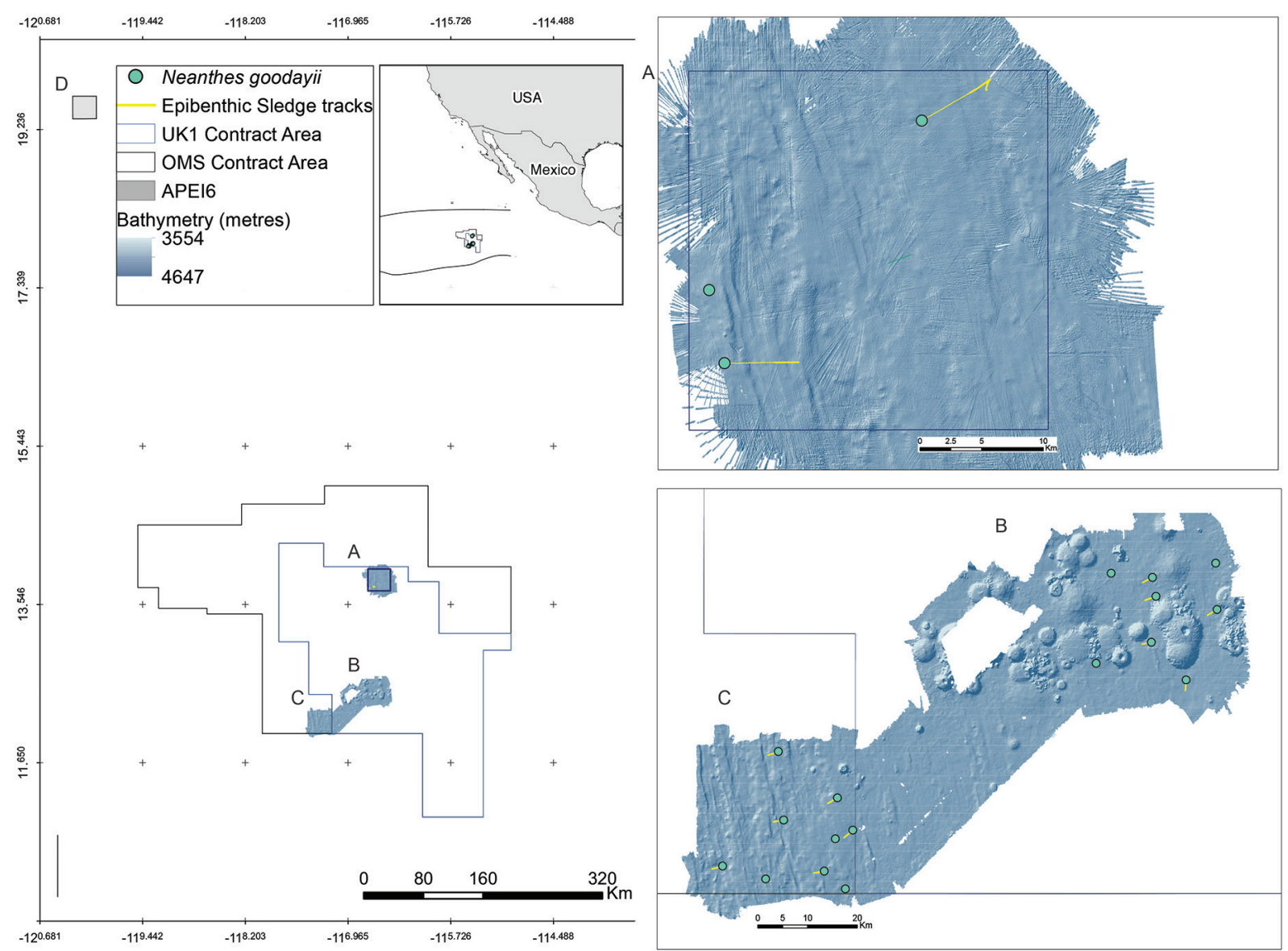

Fig. 1. Sampling sites, showing occurrences of Neanthes goodayi sp. nov. A. UK-1 Stratum-A study area within the UK Seabed Resources UK-1 exploration contract area. B. UK-1 Stratum-B study area within the UK Seabed Resources UK-1 exploration contract area. C. OMS Stratum-A study area, in the Ocean Mineral Singapore (OMS) polymetallic nodule exploration contract area. D. Area of Particular Environmental Interest APEI-6. Inset map showing location of Clarion-Clipperton Fracture Zone in the Central Eastern Pacific. Bathymetric survey data and sampling localities from the AB01 2013 RV Melville survey cruise (MV1313) and AB02 2015 RV Thomas G. Thompson survey cruise (TN319); data courtesy of Craig R. Smith (University of Hawaii), UK Seabed Resources Ltd. and Seafloor Investigations, LLC. 
Table 1. List of taxa used in phylogenetic analyses with respective NCBI GenBank accession numbers.

\begin{tabular}{|c|c|c|c|}
\hline \multirow{2}{*}{ Taxon name } & \multicolumn{3}{|c|}{ GenBank accession numbers } \\
\hline & $18 \mathrm{~S}$ & $16 S$ & COI \\
\hline Alitta succinea (Leuckart, 1847) & AY210447 & KT959483 & KT959389 \\
\hline Alitta virens (M. Sars, 1835) & Z83754 & - & AF221572 \\
\hline Ceratocephale abyssorum (Hartman \& Fauchald, 1971) & GQ426585 & GQ426618 & - \\
\hline Ceratocephale loveni Malmgren, 1867 & DQ442616 & DQ442614 & - \\
\hline Ceratonereis longiceratophora Hartmann-Schröder, 1985 & $\mathrm{AB} 106251$ & - & AY583701 \\
\hline Dendronereis aestuarina Southern, 1921 & KT900288 & - & - \\
\hline Dendronereis sp. (CUGD1) & KF586536 & - & - \\
\hline Dendronereis sp. (CUGD2) & KF586537 & - & - \\
\hline Hediste atoka Sato \& Nakashima, 2003 & LC323073 & AB703090 & AB603842 \\
\hline Hediste diadroma Sato \& Nakashima, 2003 & LC323646 & LC323062 & - \\
\hline Hediste diversicolor (O.F. Müller, 1776) & LC381864 & LC323090 & KR916844 \\
\hline Hediste japonica (Izuka, 1908) & LC323647 & LC323064 & AB603758 \\
\hline Hediste limnicola (Johnson, 1903) & LC381865 & LC323068 & - \\
\hline Namalycastis abiuma group sp. MM-2010 & HQ157237 & HM138705 & JQ081269 \\
\hline Namalycastis hawaiiensis (Johnson, 1903) & LC213729 & LC213728 & - \\
\hline Namalycastis jaya Magesh et al., 2012 & HQ157238 & HM138706 & HQ456363 \\
\hline Neanthes goodayi sp. nov. (NHM_171) & MZ408643 & MZ408646 & MZ407911 \\
\hline Neanthes goodayi sp. nov. (NHM_173) & MZ408644 & MZ408647 & MZ407912 \\
\hline Neanthes acuminata isolate $\mathrm{ABF} 1$ & - & KJ538978 & KJ539071 \\
\hline Neanthes acuminata isolate LAF1 & - & KJ538984 & KJ539083 \\
\hline Neanthes acuminata isolate NPF5 & - & KJ538994 & KJ539092 \\
\hline Neanthes acuminata isolate POF6 & - & KJ538966 & KJ539101 \\
\hline Neanthes acuminata isolate VLF1 & - & KJ538969 & KJ539128 \\
\hline Neanthes cf. glandicincta (Southern, 1921) & - & LC323071 & LC323035 \\
\hline Neanthes fucata (Savigny, 1822) & - & - & KR916874 \\
\hline Neanthes meggitti (Monro, 1931) & - & MF959006 & MF958994 \\
\hline Neanthes shinkai Shimabukuro et al., 2017 & - & - & LC331618 \\
\hline Neanthes sp. LH-2011 & - & - & JF293305 \\
\hline Neanthes wilsonchani Lee \& Glasby, 2015 & - & MF850380 & MG251655 \\
\hline Nectoneanthes oxypoda (Marenzeller, 1879) & KX290701 & - & - \\
\hline Neogyptis carriebowcayi Pleijel et al., 2012 & JN631338 & JN631325 & JN631315 \\
\hline Neogyptis fauchaldi Pleijel et al., 2012 & JN631339 & JN631326 & JN631316 \\
\hline Neogyptis hinehina Pleijel et al., 2012 & JN631340 & JN631328 & JN631317 \\
\hline Nereididae sp. (MB-2010) & GQ426586 & - & - \\
\hline Nereis heterocirrata Treadwell, 1931 & KC840697 & KC833487 & GU362684 \\
\hline Nereis pelagica Linnaeus, 1758 & AF474279 & AY340470 & HM473499 \\
\hline Nereis sandersi Blake, 1985 & AM159579 & - & - \\
\hline Nereis vexillosa Grube, 1851 & DQ790083 & GU362677 & HM473511 \\
\hline Perinereis aibuhitensis (Grube, 1878) & KC840692 & KC833485 & JX503021 \\
\hline Perinereis cultrifera (Grube, 1840) & KJ182978 & KC833495 & KR916911 \\
\hline Perinereis mictodonta (Marenzeller, 1879) & - & KC833496 & KC800632 \\
\hline Perinereis nuntia (Lamarck, 1818) & - & LC482156 & MH337359 \\
\hline Perinereis wilsoni Glasby \& Hsieh, 2006 & KC840691 & KC833494 & KC800623 \\
\hline Platynereis australis (Schmarda, 1861) & KT900290 & - & - \\
\hline Platynereis dumerilii (Audouin \& Milne Edwards, 1833) & AY894303 & KP640622 & KC591838 \\
\hline Pseudonereis sp. (pse179) & KT900283 & - & - \\
\hline Pseudonereis variegate (Grube, 1857$)$ & KC840693 & KC833493 & HQ705183 \\
\hline
\end{tabular}


DRENNAN R. et al., A new nereidid from deep-sea polymetallic nodules

(Medlin et al. 1988) and 18SB 5'-ACCTTGTTACGACTTTTACTTCCTC-3' (Nygren \& Sundberg 2003). Around 450 bp of $16 \mathrm{~S}$ rRNA (16S) were amplified using the primers ann16Sf 5'-GCGGTATCCTGACCGTRCWAAGGTA-3' (Sjölin et al. 2005) and 16SbrH 5'-CCGGTCTGAACTCAGATCACGT-3' (Palumbi 1996), and around $650 \mathrm{bp}$ of cytochrome $c$ oxidase subunit I (COI) were amplified using LCO1490 5'-GGTCAACAAATCATAAAGATATTGG-3' (Folmer et al. 1994) and COI-E 5'-TATACTTCTGGGTGTCCGAAGAATCA-3' (Bely \& Wray 2004). PCR mixtures contained $1 \mu \mathrm{l}$ of each primer $(10 \mu \mathrm{M}), 2 \mu \mathrm{l}$ template DNA and $21 \mu \mathrm{l}$ of Red Taq DNA Polymerase 1.1X MasterMix (VWR) in a total mixture of $25 \mu$. The PCR amplification profile consisted of initial denaturation at $95^{\circ} \mathrm{C}$ for $5 \mathrm{~min}, 35$ cycles of denaturation at $94^{\circ} \mathrm{C}$ for $45 \mathrm{~s}$, annealing at $55^{\circ} \mathrm{C}$ for $45 \mathrm{~s}$, extension at $72^{\circ} \mathrm{C}$ for $2 \mathrm{~min}$ and a final extension at $72^{\circ} \mathrm{C}$ for $10 \mathrm{~min}$. PCR products were purified using the Millipore Multiscreen 96-well PCR Purification System and sequencing was performed on an ABI 3730XL DNA Analyser (Applied Biosystems) at the Natural History Museum Sequencing Facility, using the same primers as in the PCR reactions plus two internal primers for 18S, 620F 5'-TAAAGYTGYTGCAGTTAAA-3' (Nygren \& Sundberg 2003) and 1324R 5'-CGGCCATGCACCACC-3' (Cohen et al. 1998). Overlapping sequence fragments were merged into consensus sequences using Geneious (Kearse et al. 2012). The sequences obtained in this study were aligned together with sequences from Genbank (Table 1) using MAFFT (Katoh 2002) for $18 \mathrm{~S}$ and 16S, and MUSCLE (Edgar 2004) for COI, both programs used as plugins in Geneious, with default settings. The $18 \mathrm{~S}$ alignment consisted of 1819 characters, $16 \mathrm{~S}$ of 514 characters and the COI alignment of 657 characters.

In total 47 terminal taxa were used in the phylogenetic analyses, with 44 from Nereididae, and three taxa from Hesionidae Grube, 1850, another family within Nereidiformia, as the outgroup. While some earlier studies suggest that Chrysopetalidae Ehlers, 1864 is sister taxon to Nereididae (Dahlgren et al. 2000), later analyses have indicated that the Nereidiformia relationships are unresolved (Weigert \& Bleidorn 2016), which justify the use of Hesionidae as the outgroup here. The program jModelTest (Posada 2008) was used to assess the best model for each partition (18S, 16S and COI) with BIC, which suggested GTR $+\mathrm{I}+\mathrm{G}$ as the best model for all genes. The data was partitioned into the three parts (18S, $16 \mathrm{~S}$ and $\mathrm{COI}$ ) and this evolutionary model was applied to each partition. The parameters used for the partitions were unlinked. Bayesian phylogenetic analyses (BAs) were conducted with MrBayes ver. 3.2.6 (Ronquist et al. 2012). Analyses were run three times for 10000000 generations. Of these, the first 2500000 generations were discarded as burn-in. Tree files were interpreted with FigTree ver. 1.4.2 (available from http://tree.bio.ed.ac.uk/software/figtree/).

\section{Data management}

The management and transfer of specimen data between a central museum database, a molecular collections database, and external data repositories and aggregators (e.g., GenBank, World Register of Marine Species (WoRMS), Ocean Biodiversity Information System (OBIS), Global Biodiversity Information Facility (GBIF), Global Genome Biodiversity Network (GGBN), and ZooBank) was carried out through the usage of DarwinCore data standards (Wieczorek et al. 2012) including the GGBN DarwinCore extensions (Droege et al. 2016). See Glover et al. (2016) for further elaboration of this data pipeline. All specimens and DNA vouchers are archived in the Natural History Museum London collections. All specimen occurrence (and associated preparation) data are provided in a DarwinCore Archive (DWcA) in the supplementary material (Supp. file 1). All mapping was carried out using ArcGIS ver. 10.2.2.

\section{Institutional abbreviations}

NHMUK = Natural History Museum, London, UK

$\mathrm{ZMH} \quad=$ Zoological Museum Hamburg 


\title{
Results
}

Phylum Annelida Lamarck, 1809

Class Polychaeta Grube, 1850

Order Phyllodocida Dales, 1962

Family Nereididae Blainville, 1818

Neanthes Kinberg, 1865

Neanthes Kinberg, 1865: 171.

Neanthes - Fauchald 1977: 89. - Wilson 1984: 210; 1988: 5. - Wu et al. 1985: 143-144. - Bakken \&

Wilson 2005: 527. — Glasby et al. 2011: 363. — Sato 2013: 35. — Ibrahim et al. 2019: 85.

\section{Type species}

Neanthes vaalii Kinberg, 1865 by subsequent designation (Hartman 1954: 27). Southern Australia.

\author{
Neanthes goodayi sp. nov. \\ urn:Isid:zoobank.org:act:C5CDA152-0C73-46BB-955F-9BD5F02BE0F6
}

Figs 2-6, 8

\section{Diagnosis}

Anterior eye pair very large, distinct, posterior eyes minute. Posterio-dorsal tentacular cirri reaching chaetigers 8-12. Two pigmented spots on dorsum of apodous segment. Palpostyles and palpophores rounded, spherical to ovoid. Paragnaths in pharangeal areas: $\mathrm{I}=1-2, \mathrm{II}=9-12$, III $=6, \mathrm{IV}=12-16$, $\mathrm{V}=0, \mathrm{VI}=1-4$, VII-VIII $=12-19$; area VI-I-VI pattern $\lambda$-shaped on oral ring. Chaetigers $1-2$ uniramous, remaining chaetigers biramous. Parapodial lobes conical, becoming narrower in posterior chaetigers. Neuracicular postchaetal lobe longer than or equal to neuraciular ligule on anterior chaetigers, shorter on medial chaetigers, papilliform or absent on posterior chaetigers. Dorsal cirri exceed length of ligules on anterior chaetigers, as long as or slightly shorter than ligules on medial chaetigers, becoming longer and exceeding ligules towards posterior end; on largest specimens, dorsal cirri exceed ligules on all chaetigers. Notochatae with homogomph spinigers throughout, supraciular nerurochaetae with homogomph spinigers and heterogomph falcigers throughout, subacicular neurochaetae with homogomph spinigers, homogomph falcigers and heterogomph falcigers throughout.

\section{Etymology}

Named in honor of Andy Gooday, member of the science party of both ABYSSLINE cruises. This etymology is part of the ABYSSLINE naming convention where all new taxon names are based on a randomised list of both crew and scientists of the two research cruises in order to recognise the team effort involved in this extensive sampling program (Wiklund et al. 2019).

\section{Material examined}

\section{Holotype}

PACIFIC OCEAN - Eastern Central Pacific, Clarion Clipperton Fracture Zone; $12.53717^{\circ} \mathrm{N}$, 116.60417 ${ }^{\circ}$ W; depth 4425 m; 20 Feb. 2015; A.G. Glover, H. Wiklund, T. Dahlgren and M. Brasier leg.; Brenke epibenthic sled, collected from epi-net; specimen guid:21b3d59f-5ec4-40da-9d65-4177e 7674f63, field ID: NHM_739, DNA voucher barcode: 0109493268, GenBank COI gene: MZ407918; NHMUK ANEA 2020.260 . 
DRENNAN R. et al., A new nereidid from deep-sea polymetallic nodules

Paratypes

PACIFIC OCEAN - Eastern Central Pacific, Clarion Clipperton Fracture Zone - 1 spec.; $13.75833^{\circ} \mathrm{N}, 116.69852^{\circ} \mathrm{W}$; depth $4080 \mathrm{~m}$; 11 Oct. 2013; A.G. Glover, H. Wiklund, T.G. Dahlgren and M.N. Georgieva leg.; Brenke epibenthic sled, collected from epi-net; specimen guid: 2d448c5f-bf70-4ed1-a541-9b505ec46434, field ID: NHM_127, DNA voucher barcode: 0109492959, GenBank 16S gene: MZ408645; NHMUK ANEA 2020.33 • 1 spec.; $13.93482^{\circ} \mathrm{N}, 116.55018^{\circ} \mathrm{W}$; depth $4082 \mathrm{~m}$; 14 Oct. 2013; same collectors and collection method as for preceding; specimen guid: f5f08fc7-49b4-446f-9f04-fbbca84f7886, field ID: NHM_171, DNA voucher barcode: 0109492952, GenBank 18S gene: MZ408643, 16S gene: MZ408646, COI gene: MZ407911; NHMUKANEA2020.34 • 1 spec.; $13.81167^{\circ} \mathrm{N}, 116.71^{\circ} \mathrm{W}$; depth $4076 \mathrm{~m}$; 16 Oct. 2013; same collectors as for preceding; USNEL box corer, collected from 0-2 cm fraction; specimen guid: fb66da6c-f627-487f-a386-3454541ad33a, field ID: NHM_238, DNA voucher barcode: 0109493276, GenBank 16S gene: MZ408648, COI gene: MZ407913; NHMUK ANEA 2020.36 • 1 spec.; $12.41628^{\circ} \mathrm{N}, 116.71485^{\circ} \mathrm{W}$; depth $4127 \mathrm{~m}$; 16 Feb. 2015; A.G. Glover, H. Wiklund, T.G. Dahlgren and M. Brasier leg.; USNEL box corer, collected from nodule; specimen guid: e1461d7d-c6c8-46fc-b951-f5ee88550a5b, field ID: NHM_512, DNA voucher barcode: 0109493273, GenBank 16S gene: MZ408651; NHMUK ANEA 2020.1 • 1 spec.; $12.53717^{\circ} \mathrm{N}$, $116.60417^{\circ} \mathrm{W}$; depth $4425 \mathrm{~m}$; $20 \mathrm{Feb} .2015$; same collectors as for preceding; Brenke epibenthic sled, collected from epi net; specimen guid: 0d2be1b6-4348-46a2-a1a7-b214562c7b18; field ID: NHM_790, DNA voucher barcode: 0109493261, GenBank 16S gene: MZ408660; NHMUK ANEA 2020.7 • 1 spec.; $12.25733^{\circ} \mathrm{N}, 117.30216^{\circ} \mathrm{W}$; depth $4302 \mathrm{~m}$; 1 Mar. 2015; same collectors and collection method as for preceding; specimen guid: bb93253e-2d66-4592-b569-cfa5976fed33, field ID: NHM 1254, DNA voucher barcode: 0109493252, GenBank 16S gene: MZ408667; NHMUK ANEA 2020.17 • 1 spec.; $12.59688^{\circ} \mathrm{N}, 116.49357^{\circ} \mathrm{W}$; depth $4258 \mathrm{~m}$; 9 Mar. 2015; same collectors as for preceding; USNEL box corer, collected from nodule; specimen guid: 333370c7-eb36-429c-96ed-fce5658f2ad2, field ID: NHM_1624, DNA voucher barcode: 0109493249, GenBank 16S gene: MZ408670;

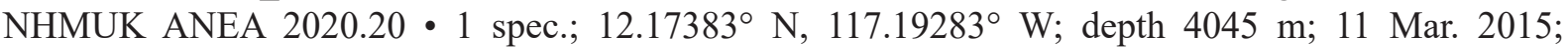
same collectors as for preceding; Brenke epibenthic sled, collected from epi-net; specimen guid: 6d7f58fc-a657-47f4-9261-7517228de6a1, field ID: NHM 1783, DNA voucher barcode: 0109493246, GenBank 16S gene: MZ408673, COI gene: MZ407927; NHMUK ANEA 2020.23 • $1 \mathrm{spec}$.; $12.02738^{\circ} \mathrm{N}$, $117.3252^{\circ} \mathrm{W}$; depth $4139 \mathrm{~m}$; 17 Mar. 2015; same collectors as for preceding; USNEL box corer, collected from nodule; specimen guid: 8abc43ad-193d-4e35-b548-6d2d0b7777f8, field ID: NHM 2069, DNA voucher barcode: 0109493237, GenBank 16S gene: MZ408681; NHMUK ANEA 2020.31.

\section{Other material}

PACIFIC OCEAN - Eastern Central Pacific, Clarion Clipperton Fracture Zone - 1 spec.; $13.93482^{\circ} \mathrm{N}, 116.55018^{\circ} \mathrm{W}$; depth 4082 m; 14 Oct. 2013; A.G. Glover, H. Wiklund, T.G. Dahlgren and M.N. Georgieva leg.; Brenke epibenthic sled, collected from epi-net; specimen guid: 022c1d2a-8b2a-479f-8ed2-20ff4e9610dd, field ID: NHM_173, DNA voucher barcode: 0109493277, GenBank 18S gene: MZ408644, 16S gene: MZ408647, COI gene: MZ407912; NHMUK ANEA $2020.35 \cdot 1 \mathrm{spec}$.; $13.81167^{\circ} \mathrm{N}, 116.71^{\circ} \mathrm{W}$; depth $4076 \mathrm{~m}$; 16 Oct. 2013 ; same collectors as for preceding; USNEL box corer, collected from $0-2 \mathrm{~cm}$ fraction; specimen guid: 57002bc8-fa3a-4a55-b823-0af978cd2fcd, field ID: NHM_239, DNA voucher barcode: 0109493275, GenBank 16S gene: MZ408649, COI gene: MZ407914; NHMUK ANEA 2020.37 • 1 spec.; same collection data as for preceding; specimen guid: 4a8718c5-d675-4044-9d2b-613fld8d5fda, field ID: NHM_240, DNA voucher barcode: 0109493274, GenBank 16S gene: MZ408650, COI gene: MZ407915; NHMUK ANEA 2020.38 • 1 spec.; 12.38624 $\mathrm{N}$, $116.54867^{\circ} \mathrm{W}$; depth $4202 \mathrm{~m}$; 17 Feb. 2015; A.G. Glover, H. Wiklund, T.G. Dahlgren and M. Brasier leg.; Brenke epibenthic sled, collected from epi-net; specimen guid: f61f9136-a39a-4696-8fdc-68aee0af5101, field ID: NHM_614, DNA voucher barcode: 0109493272, GenBank 16S gene: MZ408652, COI gene: MZ407916; NHMUK ANEA 2020.2 • 1 spec.; same collection data as for preceding; specimen guid: 1033aa6b-4093-41fc-af75-9ad090dd4c56, field 
ID: NHM_644, DNA voucher barcode: 0109493271, GenBank 16S gene: MZ408653, COI gene: MZ407917; NHMUK ANEA 2020.257 • 1 spec.; $12.53717^{\circ} \mathrm{N}, 116.60417^{\circ} \mathrm{W}$; depth $4425 \mathrm{~m}$; 20 Feb. 2015; same collectors and collection method as for preceding; specimen guid: 9a97230a-4b78-4823-88a5-d02d9c874db9; field ID: NHM_678, DNA voucher barcode: 0109493270, GenBank 16S gene: MZ408654; NHMUK ANEA 2020.258 - 1 spec.; same collection data as for preceding; specimen guid: 954c9c61-3e45-45a4-8522-7aadd1c86c60; field ID: NHM_692, DNA voucher barcode: 0109493269, GenBank 16S gene: MZ408655; NHMUK ANEA 2020.259 • 1 spec.; same collection data as for preceding; specimen guid: 76f62614-0cae-4177-8312-e231f5107f8c; field ID: NHM_743, DNA voucher barcode: 0109492976, GenBank 16S gene: MZ408656; NHMUK ANEA 2020.261 - 1 spec.; same collection data as for preceding; specimen guid: 3951d751-f1ba-44ae-8368-261047c07b12; field ID: NHM_755, DNA voucher barcode: 0109493257, GenBank COI gene: MZ407919; NHMUK ANEA 2020.3 • 1 spec.; same collection data as for preceding; specimen guid: 67a9133b-c57b-49c6-b6e4-124eb1315eac; field ID: NHM_757, DNA voucher barcode: 0109493258, GenBank 16S gene: MZ408657; NHMUK ANEA $2020.4 \cdot 1$ spec.; same collection data as for preceding; specimen guid: b13dc262-c631-44dc-927e-6a04c3608bda; field ID: NHM_766, DNA voucher barcode: 0109493259, GenBank 16S gene: MZ408658; NHMUK ANEA 2020.5 • 1 spec.; same collection data as for preceding; specimen guid: d9e557c5-3ffd-4a39-9eed-5ecead5e735f; field ID: NHM 783A, DNA voucher barcode: 0109493260, GenBank 16S gene: MZ408659; NHMUK ANEA 2020.6 - 1 spec.; same collection data as for preceding; specimen guid: 792a4c9a-9653-4ce1-8683-ca2556c1999a8; field ID: NHM_793, DNA voucher barcode: 0109493262 , GenBank COI gene: MZ407920; NHMUK ANEA 2020.8 • 1 spec.; $12.57903^{\circ} \mathrm{N}, 116.68697^{\circ} \mathrm{W}$; depth $4237 \mathrm{~m}$; 22 Feb. 2015; same collectors as for preceding; USNEL box corer, collected from $0-2 \mathrm{~cm}$ fraction; specimen guid: a933dd63-64d1-4e45-95ad-7d68282dd892; field ID: NHM_865, DNA voucher barcode: 0109493263, GenBank COI gene: MZ407921; NHMUK ANEA 2020.9 • 1 spec.; $12.57133^{\circ} \mathrm{N}, 116.6105^{\circ} \mathrm{W}$; depth $4198 \mathrm{~m}$; $23 \mathrm{Feb} .2015$; same collectors as for preceding; Brenke epibenthic sled, collected from epi-net; specimen guid: 3e7262c7-fd75-4a53-9d6c-9d01955d1bef; field ID: NHM 950, DNA voucher barcode: 0109493264, GenBank 16S gene: MZ408661, COI gene: MZ407922; NHMUK ANEA 2020.10 • 1 spec.; same collection data as for preceding; specimen guid: 06c15319-2b89-4899-b2e5-1fcd8e4a9413; field ID: NHM_971, DNA voucher barcode: 0109493265, GenBank COI gene: MZ407923; NHMUK ANEA 2020.11 • 1 spec.; $12.13367^{\circ} \mathrm{N}, 117.292^{\circ} \mathrm{W}$; depth $4122 \mathrm{~m}$; 24 Feb. 2015; same collectors and collection method as for preceding; specimen guid: 165a459f-8b81-4e97-8e82-cdcd013e1ed1; field ID: NHM_1011, DNA voucher barcode: 0109493266, GenBank 16S gene: MZ408662, COI gene: MZ407924; NHMUK ANEA 2020.12 - 1 spec.; $12.1155^{\circ} \mathrm{N}, 117.1645^{\circ} \mathrm{W}$; depth $4100 \mathrm{~m} ; 26$ Feb. 2015 ; same collectors and collection method as for preceding; specimen guid: a343e242-410a-4817-98c6-7125db7d03e7; field ID: NHM_1079, DNA voucher barcode: 0109493267, GenBank 16S gene: MZ408663; NHMUK ANEA 2020.13 • 1 spec.; same collection data as for preceding; specimen guid: 7ead0546-d0bd-4381-83af-89f58d8f8f4c; field ID: NHM_1167A, DNA voucher barcode: 0109492975, GenBank 16S gene: MZ408664; NHMUK ANEA 2020.14 - 1 spec.; same collection data as for preceding; specimen guid: 6b51d602-83f1-4bb4-b71a-e85cdbcbe8dc; field ID: NHM_1171, DNA voucher barcode: 0109493254, GenBank 16S gene: MZ408665, COI gene: MZ407925; NHMUK ANEA 2020.15 - 1 spec.; $12.00945^{\circ} \mathrm{N}, 117.17812^{\circ} \mathrm{W}$; depth $4144 \mathrm{~m}$; $27 \mathrm{Feb} .2015$; same collectors as for preceding; USNEL box corer, collected from nodule; specimen guid: 9e903864-55e8-4a1a-b532-c47af39b95f4; field ID: NHM_1194, DNA voucher barcode: 0109493253, GenBank 16S gene: MZ408666; NHMUK ANEA $2020.16 \cdot 1$ spec.; $12.45433^{\circ} \mathrm{N}, 116.61283^{\circ} \mathrm{W}$; depth $4137 \mathrm{~m}$; 3 Mar. 2015; same collectors as for preceding; Brenke epibenthic sled, collected from epi-net; specimen guid: e5797775-7141-4eb5-bb5e-dbcb29f7b42e; field ID: NHM_1480E, DNA voucher barcode: 0109493251, GenBank 16S gene: MZ408668; NHMUK ANEA 2020.18 • 1 spec.; $12.51317^{\circ} \mathrm{N}, 116.49133^{\circ} \mathrm{W}$; depth $4252 \mathrm{~m}$; 5 Mar. 2015; same collectors and collection method as for preceding; specimen guid: 35bae0ad-f00e-442b-a8f5-b1b318bf1015; field ID: NHM_1515, DNA voucher barcode: 0109493250, 
GenBank 16S gene: MZ408669, COI gene: MZ407926; NHMUK ANEA 2020.19 • 1 spec.; $12.59688^{\circ} \mathrm{N}, 116.49357^{\circ} \mathrm{W}$; depth $4258 \mathrm{~m}$; 9 Mar. 2015; same collectors as for preceding; USNEL box corer, collected from nodule; specimen guid: 29flclbf-5bca-4ed1-a893-edcd45493e04; field ID: NHM_1631A, DNA voucher barcode: 0109493248, GenBank 16S gene: MZ408671; NHMUK ANEA $2020.21 \cdot 1$ spec.; $12.17383^{\circ} \mathrm{N}, 117.19283^{\circ} \mathrm{W}$; depth $4045 \mathrm{~m}$; 11 Mar. 2015; same collectors as for preceding; Brenke epibenthic sled, collected from epi-net; specimen guid: 83507a57-c168-4b6f-b984-1c69ccbebc27; field ID: NHM_1764, DNA voucher barcode: 0109493247 , GenBank 16S gene: MZ408672; NHMUK ANEA 2020.22 • 1 spec.; $12.0999^{\circ} \mathrm{N}, 117.1966^{\circ} \mathrm{W}$; depth $4051 \mathrm{~m}$; 12 Mar. 2015; same collectors as for preceding; USNEL box corer, collected from 0-2 cm fraction; specimen guid: f79fb7b6-ed29-4cc3-9f7f-8d4ace75c585; field ID: NHM_1836A, DNA voucher barcode: 0109493245, GenBank 16S gene: MZ408674; NHMUK ANEA 2020.24 • 1 spec.; $12.0415^{\circ} \mathrm{N}, 117.21717^{\circ} \mathrm{W}$; depth $4094 \mathrm{~m}$; 13 Mar. 2015; same collectors as for preceding; Brenke epibenthic sled, collected from epi-net; specimen guid: 0508d326-ef73-4f52-bdc6-757b2ab745fe; field ID: NHM_1866, DNA voucher barcode: 0109492983, GenBank 16S gene: MZ408675, COI gene: MZ407928; NHMUK ANEA 2020.25 • 1 spec.; same collection data as for preceding; specimen guid: 922ad1d7-bd75-4588-ba2e-be32cfe432c5; field ID: NHM_1891, DNA voucher barcode: 0109492960, GenBank 16S gene: MZ408676; NHMUK ANEA 2020.26 • 1 spec.; same collection data as for preceding; specimen guid: e991eafe-0593-4e08-8967-d77e017eabac; field ID: NHM_1929A, DNA voucher barcode: 0109493233, GenBank 16S gene: MZ408677, COI gene: MZ407929; NHMUK ANEA 2020.27 • 1 spec.; same collection data as for preceding; specimen guid: 62b28de1-a797-4ec0-99cf-e38625b0e01c; field ID: NHM 1929B, DNA voucher barcode: 0109493234, GenBank 16S gene: MZ408678; NHMUK ANEA 2020.28 - 1 spec.; same collection data as for preceding; specimen guid: 25953aef-8a48-48d1-9fc2-b0a86ec7d052; field ID: NHM_1947D, DNA voucher barcode: 0109493235, GenBank 16S gene: MZ408679; NHMUK ANEA 2020.29 • 1 spec.; $12.0505^{\circ} \mathrm{N}, 117.40467^{\circ} \mathrm{W}$; depth $4235 \mathrm{~m}$; 16 Mar. 2015; same collectors and collection method as for preceding; specimen guid: d8edb41d-51d6-4fbd-a547-92fa290209d4; field ID: NHM 2014, DNA voucher barcode: 0109493236, GenBank 16S gene: MZ408680, COI gene: MZ407930; NHMUK ANEA $2020.30 \cdot 1 \mathrm{spec} . ; 1^{2} .57133^{\circ} \mathrm{N}, 116.6105^{\circ} \mathrm{W}$; depth $4198 \mathrm{~m} ; 23 \mathrm{Feb} .2015$; same collectors as

for preceding; Brenke epibenthic sled, collected from supra-net; specimen guid: 1c30624d-19a0-43f0-92dc-9a315a3e43fc; field ID: NHM 3074, DNA voucher barcode: 0109493238 , GenBank 16S gene: MZ408682; NHMUK ANEA 2020.32.

\section{Comparative material examined}

Holotype of Neanthes heteroculata (Hartmann-Schröder, 1981)

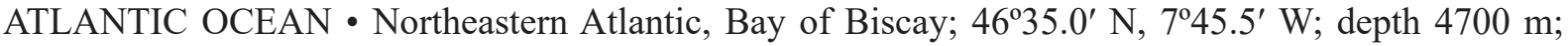
24 Oct. 1967; ZMH P-16464.

Paratypes of Neanthes heteroculata (Hartmann-Schröder, 1981)

ATLANTIC OCEAN • 2 specs; same collection data as for preceding; ZMH P-16465.

\section{Description}

Holotype (NHM_739) complete, TL $=12 \mathrm{~mm}, \mathrm{~L} 15=4.7 \mathrm{~mm}, \mathrm{~W} 15=0.9 \mathrm{~mm}$, for 47 chaetigers. Body somewhat 'baseball bat-shaped', wide, swollen anteriorly but tapering gradually posteriorly (Fig. 2A-B). Live specimen pale, iridescent and semi-translucent, with yellow gut and red blood vessels visible through body wall (Fig. 2A, C); specimen in ethanol opaque, pale beige, with some red vasculature still visible (Fig. 2B, D). Two pigmented spots on either side of dorsum of apodous segment visible in both live specimens and in ethanol, with some pigmentation also visible on dorsum of anterodorsal tentacular cirrophores (Fig. 2C-D). 

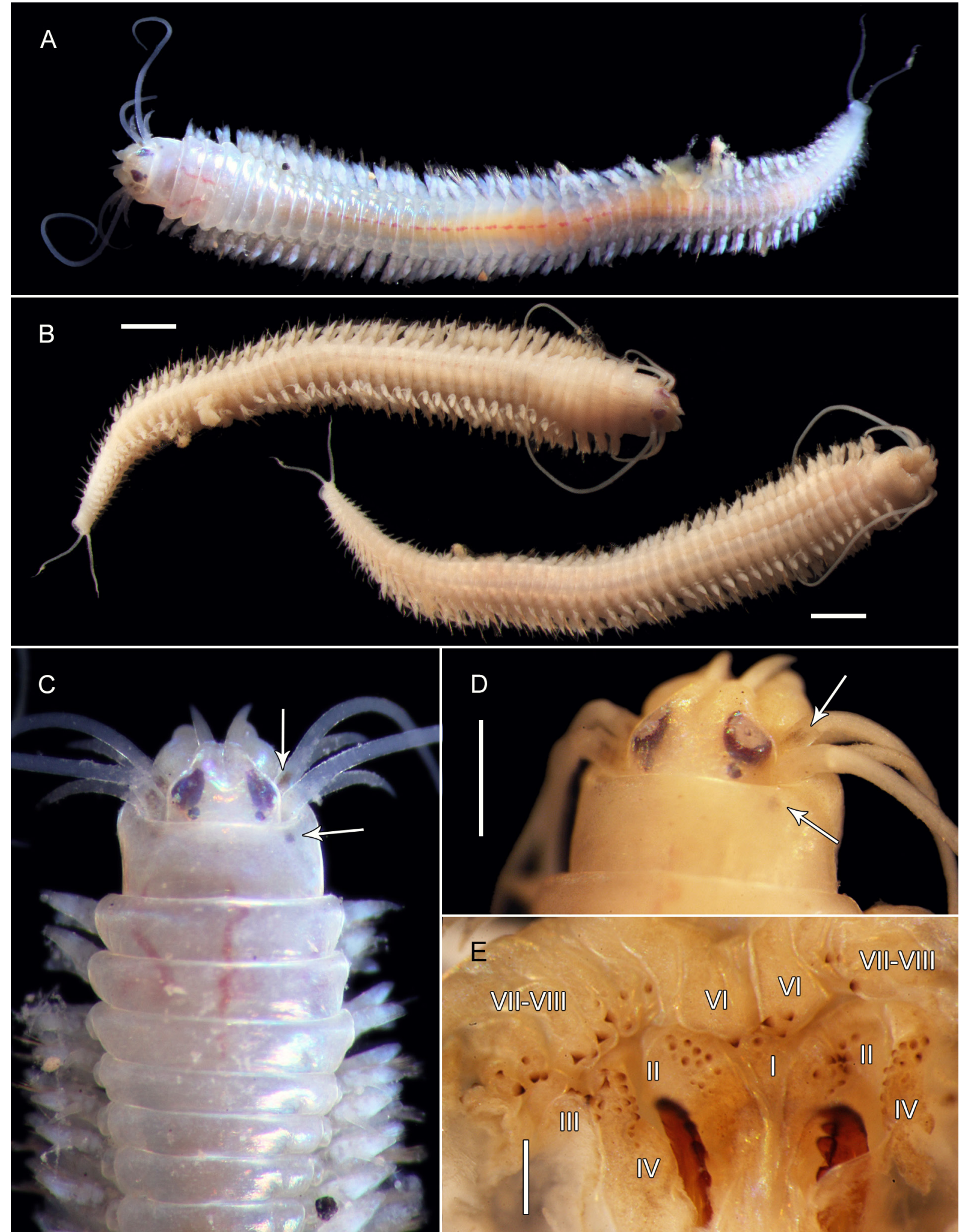

Fig. 2. Neanthes goodayi sp. nov., holotype (NHM_739). A. Live image, entire specimen. B. Preserved entire specimen, dorsal view (left), ventral view (right). C. Live image, anterior view, arrows mark pigmentation. D. Preserved specimen, anterior view, arrows mark pigmentation. E. Dissected pharynx, with pharyngeal areas I, II, III, IV, VI, VII-VIII highlighted. Scale bars: $\mathrm{B}=1 \mathrm{~mm}$; $\mathrm{D}=500 \mu \mathrm{m}$; $\mathrm{E}=250 \mu \mathrm{m}$. 
Prostomium short, rounded trapezoid with shallow dorsal depression extending anteriorly from midpoint to distal margin (Fig. 2C-D); antennae cirriform, medium-sized, barely extending beyond palps. Palps nearly as long as prostomium, with both palpophores and palpostyles short, spherical, with palpostyles half as long as palpophores. Tentacular cirri with short, cylindrical cirrophores; posterior-dorsal pair of tentacular cirri longest, extending to chaetiger 12 (Fig. 2A-B). Two pairs of dark red eyes; anterior pair very large, rounded teardrop-shaped, with large, rounded lenses inserted anterolaterally and with an irislike structure visible in preserved specimen (Fig. 2C); posterior pair of eyes minute, rounded, with small anterolateral lenses. Apodous anterior segment collar-like, slightly longer and narrower than chaetiger 1 .

Pharynx not everted. Jaws dark red-brown with 6 lateral teeth; All paragnaths brown, conical, arranged as follows (Fig. 2E): area I: 2, one large cone, one smaller cone distally; area II: 12 in cluster; area III: approx. 6 (area damaged), four cones in row with two smaller cones laterally; area IV: 13 in teardropshaped cluster, with curved line of cones extending from jaws posteriorly, ending in cluster of 7 cones; area V: no paragnaths; area VIa: 1; area VIb: 4, one large and three smaller cones in trapezoid arrangement; areas VII-VIII: 19, eight large cones in a single well-spaced row with 11 smaller cones scattered laterally. Areas VI-V-VI with $\lambda$-shaped ridge pattern.

Chaetigers 1 and 2 uniramous, with all subsequent chaetigers biramous.

Dorsal cirri inserted at base of median and dorsal ligule in uniramous and biramous chaetigers, respectively, slightly inflated on uniramous chaetigers (Fig. 3A), more slender from chaetiger 3 onwards (Fig. 3B-H); dorsal cirri extending beyond median ligule on anteriormost chaetigers (Fig. 3A-B), as long as or slightly shorter than median ligules from chaetiger 6 onwards (Fig. 3C-D) and extending beyond median ligules from around chaetiger 29 (Fig. 3E), up to twice as long as median ligules on posterior chaetigers from chaetiger 40 (Fig. $3 \mathrm{G}-\mathrm{H}$ ).

Dorsal ligule conical throughout, slightly shorter than median ligules on anterior chaetigers (Fig. 3B-C), approximately two-thirds the length of median ligules from chaetiger 10 onwards. Dorsal and median ligules reduced in size on posterior chaetigers from chaetiger 40, with dorsal ligule vanishing in posteriormost chaetigers (Fig. 3H). Median ligule slightly inflated on uniramous chaetigers (Fig. 3A), conical on biramous chaetigers, narrower from chaetiger 29 (Fig. 3E), bluntly conical on posteriormost chaetigers (Fig. 3H). Notopodial prechaetal lobe indistinct.

Neuracicular ligule shorter than ventral neuropodial ligule on anterior chaetigers (Fig. 3A-C), becoming equal in length or slightly shorter from chaetiger 10, equal or slightly longer from chaetiger 29 (Fig. 3E). Superior neuropodial lobe indistinct, truncate throughout; inferior lobe short, rounded on anterior and medial chaetigers, gradually shortening, giving neuracicular ligule pointed appearance on posterior chaetigers (Fig. 3G-H). Neuracicular prechaetal lobe indistinct. Neuracicular postchaetal lobe conical, longer than neuracicular lobe on anteriormost chaetigers (Fig. 3A-B), equal in length at chaetiger 6 (Fig. 3C), gradually shortening and becoming more digitiform on subsequent chaetigers to papilliform nub around chaetiger 29 (Fig. 3F), absent in posterior chaetigers from around chaetiger 40.

Ventral neuropodial ligule conical throughout, gradually narrowing on medial (Fig. 3E) and posterior chaetigers (Fig. 3G-H). Ligule sub-equal in length to median ligule in anterior and early medial chaetigers (Fig. 3A-D), becoming shorter in remaining chaetigers from chaetiger 29 (Fig. 3E), to two-thirds as long as ligule from chaetiger 40 (Fig. $3 \mathrm{G}$ ) and half as long on posteriormost chaetigers (Fig. 3H).

Ventral cirri cirriform (Fig. 3C-F), inserted basally to ventral neuropodial ligule throughout, slightly shorter than ligule on anterior and medial chaetigers, subequal in length on posteriormost chaetigers (Fig. 3F). 


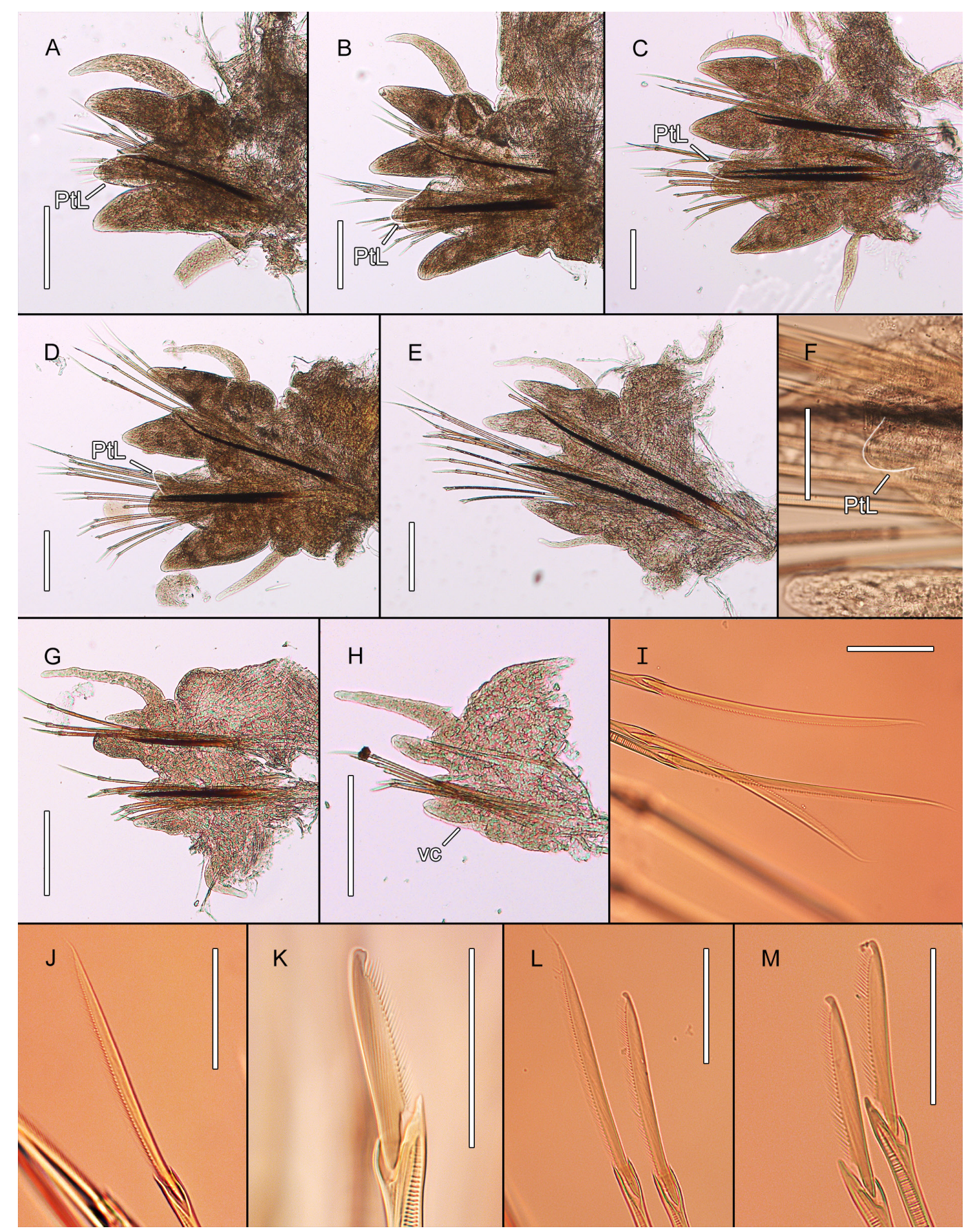

Fig. 3. Neanthes goodayi sp. nov., holotype (NHM_739). A. Chaetiger 1, posterior view. B. Chaetiger 3, posterior view. C. Chaetiger 6, posterior view. D. Chaetiger 20, posterior view. E. Chaetiger 29, posterior view. F. Chaetiger 29, posterior view, detail of neuracicular postchaetal lobe. G. Chaetiger 40, posterior view. H. Chaetiger 46, posterior view. I. Notochaetae, detail of homogomph spinigers, chaetiger 20. J. Supraciular neurochaetae, detail of homogomph spiniger, chaetiger 3. K. Supracicular neurochaetae, detail of heterogomph falciger, chaetiger 10. L. Subacicular neurochaetae, detail of homogomph spiniger (left) and homogomph falciger (right), chaetiger 20. M. Subacicular neurochaetae, detail of heterogomph falcigers, chaetiger 20. Abbreviations: $\mathrm{PtL}=$ postchaetal lobe; $\mathrm{VC}=$ ventral cirrus. Postchaetal lobe in A-D, F outlined with a fine white line. Parapodia in C, E-H dissected from left side of specimen; parapodia in $\mathrm{A}-\mathrm{B}, \mathrm{D}$ dissected from right side of specimen, with images laterally inverted follow direction of other plates. Scale bars: A-E, G-H $=200 \mu \mathrm{m} ; \mathrm{F}, \mathrm{I}-\mathrm{M}=50 \mu \mathrm{m}$. 
DRENNAN R. et al., A new nereidid from deep-sea polymetallic nodules

Pygidium somewhat pyriform, truncate distally, with two filamentous anal cirri attached ventro-laterally, extending 8 chaetigers in length (Fig. 2A-B).

Caecal glands present, small, white, slightly thickened.

Multiple aciculae per parapodial lobe observed on some chaetigers in holotype: double neuraciculae in chaetigers 2, 3, 6 and 20 (Fig. 3B-D), and triple notoaciculae on chaetiger 6 (Fig. 3C). This feature was not observed in parapodial dissections from paratypes.

Notochaetae all homogomph spinigers with long blades, of similar width towards toothed edge but drastically slendering to an aristate distal end (Fig. 3I); 4 present in anterior chaetigers, 5 in medial chaetigers, 3 in posterior chaetigers and absent from chaetiger 46 .

Supracicular neurochaetae with homogomph spinigers and heterogomph falcigers, both types present in all falcigers except final two chaetigers, where supracicular falcigers are absent. Homogomph spinigers similar in appearance to those of notopodia (Fig. 3J), though with blades reducing in length moving ventrally (shortest blades two-thirds as long as longest blade), numbering 4 on first two chaetigers, $3-5$ on anterior and medial chaetigers and 2 on posterior chaetigers where fascicles remain. Heterogomph falcigers with knob-like tips (Fig. 3K) and blades roughly half the length of shortest spinigers, numbering 1 on anterior chaetigers, 2 on medial chaetigers and 1 on posterior chaetigers where fascicles remain.

Subacicular neurochaetae with homogomph spinigers and both homogomph and heterogomph falcigers. Homogomph spinigers also similar in appearance to those of notopodia (Fig. 3L) but with blades twothirds as long and numbering 1-2 on all chaetigers. Homogomph falcigers with knob-like tips (Fig. 3L), blades three-quarters the length of spinigers (Fig. 3L), numbering 1-3 on all chaetigers. Heterogomph falcigers similar in appearance to those of supracicular fascicles (Fig. 3M), numbering 3 on first two chaetigers, $4-6$ on anterior, $2-4$ on medial and $2-3$ on posterior chaetigers.

\section{Variations}

Largest specimen (paratype NHM_2069) damaged, in two parts, TL $=17 \mathrm{~mm}$, L15 $=6.7 \mathrm{~mm}$, $\mathrm{W} 15=1 \mathrm{~mm}$ for 55 chaetigers. Smallest specimen (paratype NHM_127) with TL $=1 \mathrm{~mm}$ for 10 chaetigers (see Juveniles section below). Pigment spots on dorsum as in holotype, consistent across most specimens both live and preserved (Fig. 4A-D), pigmentation on tentacular cirrophores more variable. Palpophores spherical to ovoid in shape (e.g., Fig. 4B). Posterior-dorsal pair of tentacular cirri extending to chaetiger 8-12 in most specimens (max. chaetiger 6 in juveniles). Eyes dark red to purple, anterior pair ranging from circular/ovoid (Fig. 4B-D) to teardrop-shaped concave discs or deeper cups (Figs 4A, 5A), becoming more crescent-shaped with decreasing size (Fig. 5B-D); posterior pair mostly circular (Fig. 4A-B), but occasionally oblong (Fig. 4A) or seeming to fuse with anterior pair (Fig. 6A-B), or with one missing (Fig. 4D). Posterior eye pair often less distinct in smaller specimens (Fig. 5A-B), becoming tiny spots (Fig. 5A) or patchy and irregularly shaped (Fig. 5B), completely absent in smallest specimens (Fig. 5C-D), with trace of lens not obvious. Apodous anterior segment longer and narrower than chaetiger 1, as in holotype, to similar in length and width as chaetiger 1 (Fig. 4A-D).

Jaws with 6-7 lateral teeth; paragnaths in pharangeal areas in non-holotype specimens: $\mathrm{I}=1-2$, $\mathrm{II}=9-12, \mathrm{III}=6, \mathrm{IV}=12-16, \mathrm{~V}=0, \mathrm{VI}=2-3, \mathrm{VII}-\mathrm{VIII}=12-17$ (8 large cones in a row as in holotype, varying number of smaller cones scattered laterally). Only one specimen (epitoke male, paratype NHM_1783) with pharynx everted (Fig. 6B).

In largest specimen, dorsal cirrus exceeds median ligule on all chaetigers, neuracicular ligule remains slightly longer than ventral ligule on median and posterior chaetigers, prechaetal lobe remains as 
visible papilliform process on posterior chaetigers, ventral ligule subequal to ventral ligule from medial chaetigers onwards and ventral cirri longer than ventral ligule on posteriormost chaetigers.

Numbers of chaetae greater for most fascicles in largest specimen: notochaetae 6 homogomph spinigers on anterior and medial chaetigers, 4 in posterior chaetigers, 1 in posteriormost chaetigers; supracicular neurochaetae with 5-7 homogomph spinigers on first two chaetigers, 2-4 on anterior and medial chaetigers, 1 on posterior chaetigers, heterogomph falcigers 3 on first two chaetigers, 4-6 on anterior chaetigers, $0-3$ on medial chaetigers and 1 on posterior chaetigers; subacicular neurochaetae with 2-4 homogomph spinigers most chaetigers, 1 on posteriormost chaetigers, homogmph falcigers $3-5$ on
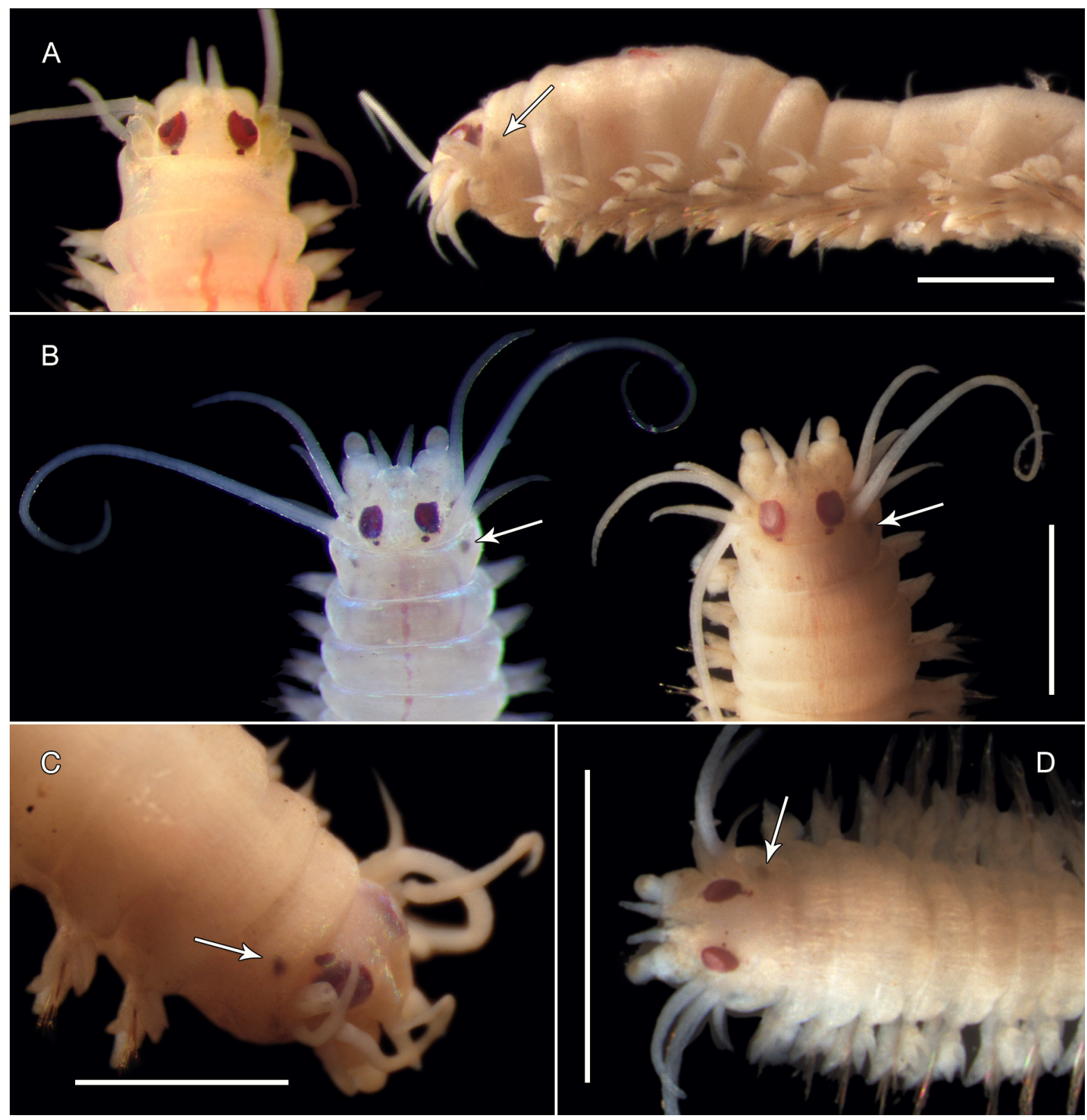

Fig. 4. Neanthes goodayi sp. nov., paratypes. A. Paratype (NHM_1624), preserved specimen; dorsal anterior view, live image (left); lateral anterior view, preserved specimen (right). B. Paratype (NHM_755); dorsal anterior view, live image (left), preserved specimen (right); arrows marking pigmentation. C. Paratype (NHM_238), dorsal anterior view, arrows mark pigmentation. D. Paratype (NHM_512), dorsal anterior view, arrows mark pigmentation. Scale bars $=1 \mathrm{~mm}$. 
anterior chaetigers, 1-2 on medial chaetigers, 1 on posterior chaetigers, heterogomph falcigers 6-9 on anterior chaetigers, $1-3$ on medial and posterior chaetigers.

\section{Description of epitoke paratype}

One epitokous specimen observed (paratype NHM_1783) (Fig. 6A). Specimen moderately damaged, posteriorly incomplete, $\mathrm{TL}=10 \mathrm{~mm}, \mathrm{~L} 15=4 \mathrm{~mm}$, for 37 chaetigers (chaetiger 15 damaged, width at chaetiger 14 excluding parapodia $0.8 \mathrm{~mm}$ ). Body divided into two regions: pre-natatory with 14 chaetigers and natatory with at least 23 chaetigers; post-natatory region unknown. Eyes not notably modified (Fig. 6A-B); anterior pair with iris-like structure as in holotype, posterior pair somewhat fused to anterior pair.

Pre-natatory chaetigers with modified dorsal and ventral cirri on chaeigers 1-7; notably thickened, but with distalmost tip remaining fine and cirriform (Fig. 6C). Chaetal types in pre-natatory chaetigers as in holotype.

Natatory chaetigers with distinctly enlarged, elongate modified parapodia (Fig. 6D). Noto- and neuropodia elongated basally, with ligules and lobes not significantly larger than on non-modified parapodia. Neuracicular ligule with lamellar structure distally. Both dorsal and ventral cirri notably elongate, with a pair of conical lobes emerging from the upper and lower base of each cirrus, not present on anterior chaetigers; dorsal cirri slightly papillated (Fig. 6D-E). Both notopodial and neuropodial fascicles dense, up to 40 chaetae per fascicle, and with only a single chaetal type: long, simple sesquigomph spinigers
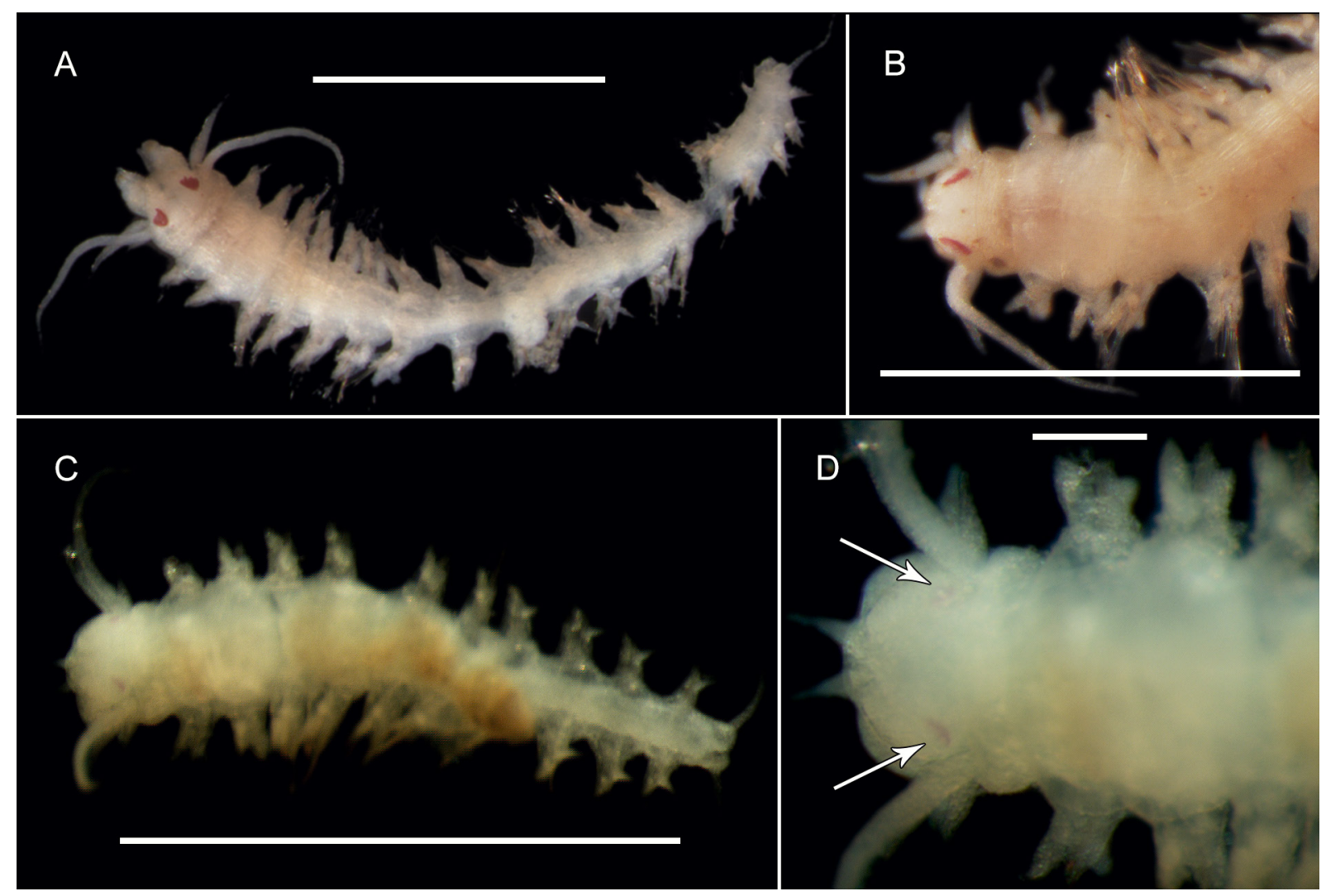

Fig. 5. Neanthes goodayi sp. nov., juvenile specimens. A. Paratype (1254), entire specimen, dorsal view. B. Paratype (NHM_171) dorsal anterior view. C. Paratype (NHM_127) entire specimen, dorsal view. D. Paratype (NHM_127), close up of dorsal anterior, arrows mark position of anterior eye pair. Scale bars: $\mathrm{A}, \mathrm{C}=1 \mathrm{~mm} ; \overline{\mathrm{B}}=500 \mu \mathrm{m} ; \mathrm{D}=100 \mu \mathrm{m}$. 

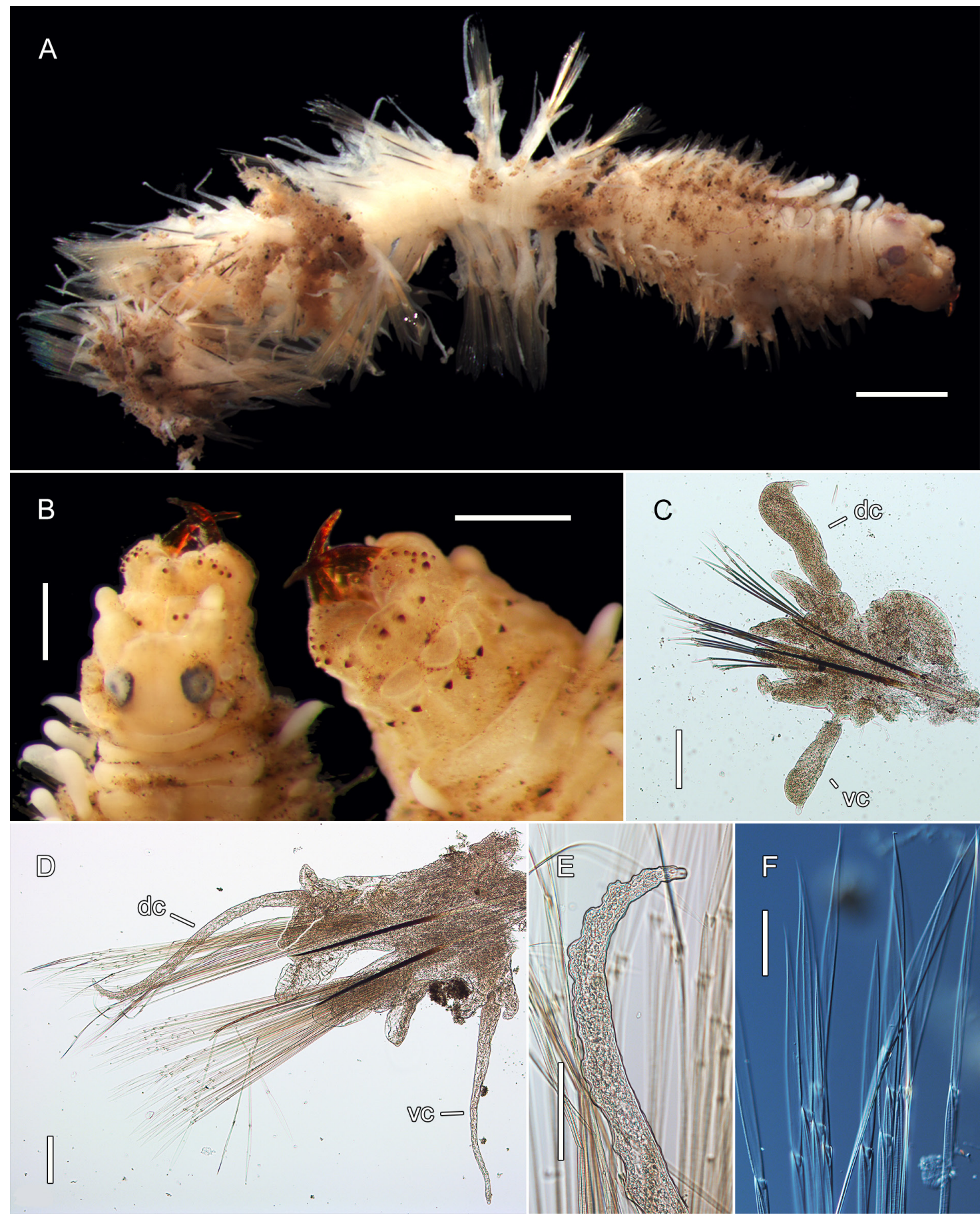

Fig. 6. Neanthes goodayi sp. nov., epitoke paratype (NHM_1783), preserved specimen. A. Entire specimen, dorsal view. B. Extruded pharynx, dorsal view (left), ventral view (right). C. Detail of prenatatory parapodium 4, with modified dorsal and ventral cirri, posterior view. D. Detail of modified natatory swimming parapodium, chaetiger 31, posterior view. E. Detail of papillated dorsal cirrus, chaetiger 31, posterior view. F. Modified swimming spinigers, subacicular neurocheatal fascicle, chaetiger 32. Abbreviations: $\mathrm{DC}=$ dorsal cirrus; $\mathrm{VC}=$ ventral cirrus. Lobe at base of dorsal cirrus in $\mathrm{D}$ and $\mathrm{E}$ outlined with a fine white or black line. Parapodium in $\mathrm{C}$ dissected from left side of specimen; parapodium in $\mathrm{D}$ dissected from right side of specimen, with images $\mathrm{D}$ and $\mathrm{E}$ laterally inverted to follow direction of other plates. Scale bars: $A=1 \mu \mathrm{mm} ; \mathrm{B}=500 \mu \mathrm{m} ; \mathrm{C}-\mathrm{D}=200 \mu \mathrm{m}$; $\mathrm{E}=100 \mu \mathrm{m} ; \mathrm{F}=50 \mu \mathrm{m}$. 
with ensiform (knife-shaped) blades (Fig. 6F). No gametes observed, though the presence of slightly papillated dorsal cirri on natatory chaetigers suggests that this specimen is a male ( $\operatorname{Read} 2007)$.

\section{Juveniles}

Several small, possibly juvenile specimens were observed; paratypes NHM_127, NHM_171, NHM_1254, TL $=1.0-2.5 \mathrm{~mm}, \mathrm{~L} 15=\max .2 .2 \mathrm{~mm}, \mathrm{~W} 15=\max .0 .2 \mathrm{~mm}, 10-18$ chaetigers (Fig. 5A-D). Posterio-dorsal tentacular cirri extending to chaetiger 6. Eyes poorly developed in these specimens, with anterior eye pair observed only as faintly pigmented crescents (Fig. 5B-D), lenses not obvious; posterior eye pair not visible in smallest specimens (Fig. 5C-D). The identity of these specimens was confirmed with genetic data. Due to their size and the delicate nature of specimens, pharyngeal and parapodial dissections were not conducted to preserve specimen integrity.

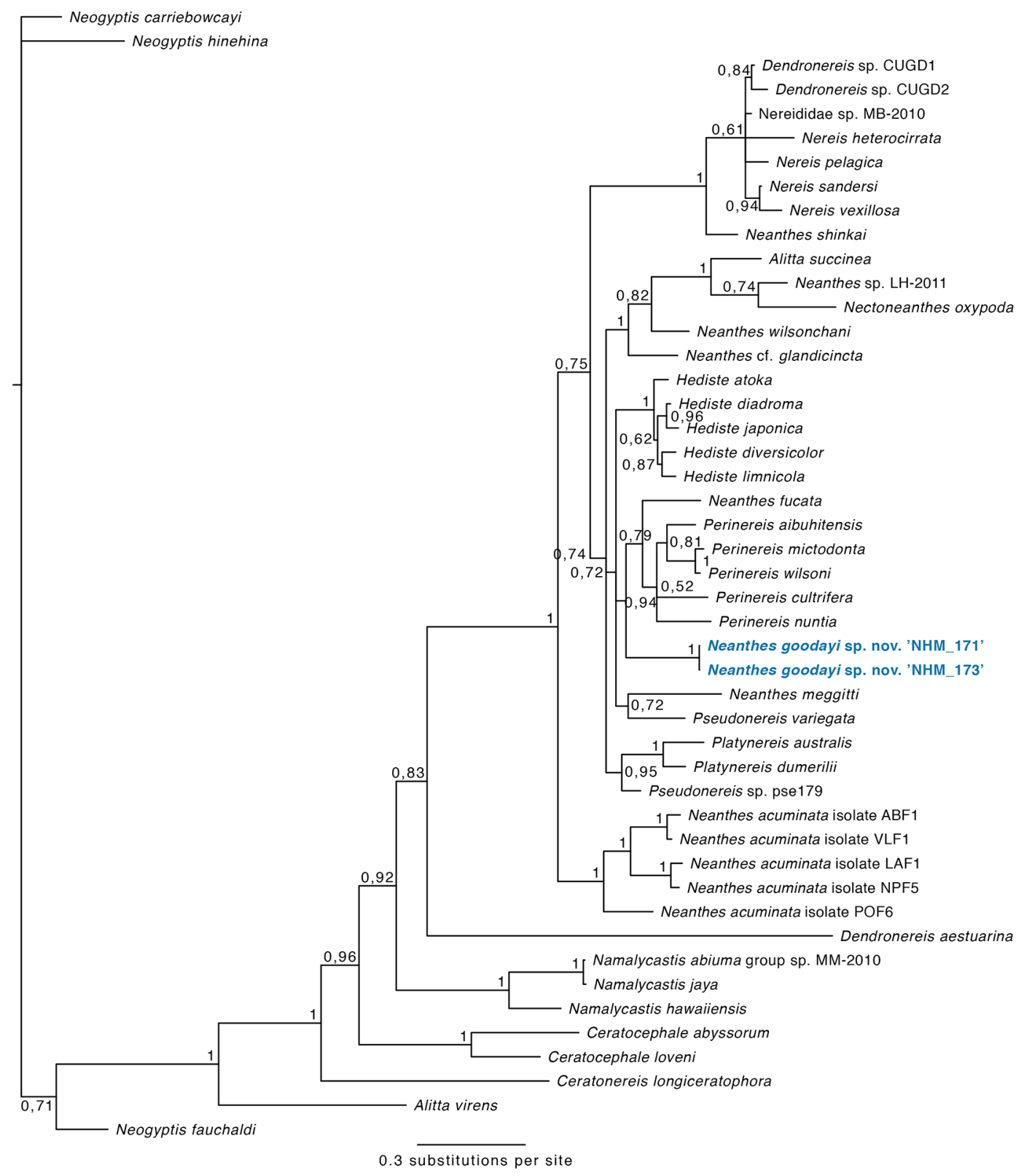

Fig. 7. Phylogenetic analysis of Nereididae Blainville, $1818,50 \%$ majority rule tree from the Bayesian analyses using $18 \mathrm{~S}, 16 \mathrm{~S}$ and COI, with posterior probability values on nodes. Forty-five taxa from GenBank were included, using three taxa from another family within Nereidiformia, Hesionidae Grube, 1850 , as outgroup. 


\section{Genetic data}

All 43 individuals were sequenced for $16 \mathrm{~S}$ and COI. The gene 16S was successfully sequenced in all but six specimens. COI sequencing was less successful; however, each specimen had coverage of at least one of the two genes. All specimens formed a single clade with low intraspecific divergence. Several specimens were also sequenced for $18 \mathrm{~S}$ in order to assess deeper taxonomic relationships. This species was genetically distinct from all other species included in our phylogenetic analyses, and forms the basal branch of a clade including Neanthes fucata (Savigny, 1822) and five species of Perinereis Kinberg, 1865 (Fig. 7).

\section{Remarks}

This species is most consistent with the genus Neanthes Kinberg, 1865, most recently defined by Ibrahim et al. (2019). Previous analyses based on morphological parsimony suggested that neither of the three most species-rich nereidid genera, Neanthes, Nereis and Perinereis, can be considered monophyletic, with many generic characters displaying high homoplasy (Bakken \& Wilson 2005). Molecular phylogenetic analyses carried out in this study supported the polyphyly of Neanthes, as sequences of species currently regarded as Neanthes, both from the ABYSSLINE material and from GenBank, rarely grouped together and were evenly distributed throughout a tree that included 11 other nereidid genera.

Neanthes goodayi sp. nov. can be differentiated from the majority of its congeners by the notably large anterior pair of eyes. Only N. heteroculata (Hartmann-Schröder, 1981), described from abyssal $(4700 \mathrm{~m})$ waters off the Bay of Biscay in the northeastern Atlantic, appears to possess comparably large anterior and minute posterior pairs of eyes. Neanthes heteroculata and $N$. goodayi sp. nov. also display similarities with regard to several other characters, such as the appearance of the prostomium, antennae and tentacular cirri, in addition to the types of chaetae present and their appearance and arrangement. Based on an examination of the type material of $N$. heteroculata, $N$. goodayi sp. nov. differs in having distinctly rounded, spherical to ovoid palpophores (e.g., Fig. 4B), with palpophores in $N$. heteroculata found to be narrower, bluntly conical in shape. Furthermore, the dorsal cirri are relatively short in $N$. heteroculata, not exceeding the length of the notopodial ligules, whereas they exceed the length of the notopodial ligules in at least anterior and posterior chaetigers in N. goodayi sp. nov.

Notably, $N$. heteroculata is one of a handful of species of Neanthes reported from the deep sea. Of the 84 currently valid species of Neanthes (Read \& Fauchald 2020b) only 13 have been reported from depths greater than $200 \mathrm{~m}$ (Khlebovich 1996; Shimabukuro et al. 2017; Hsueh 2019). Of these, N. goodayi sp. nov. also resembles $N$. papillosa (Day, 1963), described from deep $(2745 \mathrm{~m})$ waters off Cape Town, South Africa. Neanthes papillosa similarly possesses an enlarged anterior pair of eyes relative to the posterior pair, in addition to long tentacular cirri, relatively elongate, conical parapodial ligules, and dorsal cirri that exceed the length of the notopodial ligules, becoming longer on posterior chaetigers. The holotype of $N$. papillosa is noted to have pale, poorly chitinised paragnaths, thus making them difficult to observe (Day 1963). However, despite having fewer paragnaths in number across all areas, they appear to be organised in similar arrangements as in $N$. goodayi sp. nov., such as a single row of paragnaths on areas VII-VIII (single row of large cones in $N$. goodayi sp. nov. with varying numbers of smaller cones laterally). However, $N$. papillosa can primarily be differentiated from $N$. goodayi sp. nov. in that the anterior pair of eyes does not appear to be as strikingly large as in N. goodayi sp. nov. or $N$. heteroculata; thus, there is less disparity between the anterior and posterior eye pairs in size. Additionally, $N$. papillosa can be further distinguished in that it does not bear homogomph falcigers and that parapodial lobes of midbody and posterior chaetigers bear numerous club-shaped papillae; however, it is worth considering that some characters of $N$. papillosa may be reproductive modifications, as the holotype is described from a single epikotous female specimen. 
DRENNAN R. et al., A new nereidid from deep-sea polymetallic nodules

Neanthes goodayi sp. nov. also bears similarities to N. vitiazi Khlebovich, 1996 from abyssal waters (3342-4160 m) of southern Japan, primarily in terms of broadly similar paragnath distributions, bearing homogomph falcigers and in having a large anterior pair of eyes, which are illustrated as rings without strong pigment. Neanthes vitiazi differs in that it has long, digitate median ligules positioned at right angles to the notoacicula on midbody and posterior chaetigers. Neanthes vitiazi is also described as having brown pigmentation on parapodial appendages and dense spot-like pigmentation on the apodous anterior segment; $N$. goodayi sp. nov. similarly bears two pigmented spots on the dorso-lateral anterior margin of this segment; however, these are relatively small, whereas the spots in Neanthes vitiazi span much of the length of the segment and are placed dorsally, behind the eyes.

The geographically most proximal deep-water species, N. mexicana Fauchald, 1972, described from abyssal waters off Baja California, and N. sandiegensis Fauchald, 1977 from the San Diego Trough $(728-855 \mathrm{~m})$, can also be differentiated from $N$. goodayi sp. nov. Neanthes mexicana was originally described from a single damaged specimen, re-examined and revised by de León-González \& SolísWeiss (2000) with the addition of several nereidids collected from abyssal waters off California USA agreeing with the type specimen. Neanthes mexicana is described as bearing a single pair of very large red eyes, with diffuse pigment spots posterior to the eyes noted to perhaps represent the posterior eye pair (Fauchald 1972). In ABYSSLINE specimens, the appearance of the posterior eye pair was variable, ranging from discrete dark spots to more faint, irregular shapes, occasionally with one or both eyes absent all together, particularly in smaller specimens. The eye morphology of $N$. mexicana therefore falls within the variation observed in the ABYSSLINE samples. Neanthes mexicana and N. goodayi sp. nov. also share similarities in terms of parapodial morphology, with all parapodial ligules broadly conical to somewhat triangular in shape (see de León-González \& Solís-Weiss 2000: fig. 3). However, $N$. mexicana differs from $N$. goodayi sp. nov. in terms of palp morphology (long, digitate palpostyles), the arrangement and number of paragnaths (4 cones in areas II and IV versus 12 cones in both areas in $N$. goodayi sp. nov.,) and in lacking homogomph falcigers.

Neanthes sandiegensis is only known from a single damaged specimen. However, it differs from $N$. goodayi sp. nov. primarily in terms of parapodial morphology, bearing large, foliose dorsal notopodial ligules with medially inserted, long, flattened digitate dorsal cirri, long digitate prechaetal notopodial lobes and notably elongate ventral neuropodial ligules. Neanthes sandiegensis also differs in terms of the distribution and number of paragnaths on most pharyngeal areas $(I=0, I I=2, \mathrm{VI}=6-8, \mathrm{VII}-\mathrm{VIII}=35$ in $N$. sandiegensis, I $=2, \mathrm{II}=12, \mathrm{VI}=1-4, \mathrm{VIII}-\mathrm{VIII}=19$ in the holotype of $N$. goodayi sp. nov.).

While none of the morphologically most similar or geographically proximal congeners had genetic data available for comparison, morphological differences existed in each case. Neanthes goodayi sp. nov. can be differentiated from other deep-water Neanthes spp. primarily in terms of eye morphology: $N$. articulata Knox, 1960, N. donggungensis Hsueh, 2019, N. kerguelensis (McIntosh, 1885) and $N$. suluensis Kirkegaard, 1995 bear two relatively small, subequal eye pairs, whereas $N$. bioculata (Hartmann-Schröder, 1975) bears a single pair of small eyes; N. abyssorum Hartman 1967, N. kermadeca (Kirkegaard, 1995), N. shinkai Shimabukuro et al., 2017 and N. typhla (Monro, 1930) are recorded as lacking eyes altogether and can be further differentiated from $N$. goodayi sp. nov. in terms of paragnath distribution, among other characters (see Shimabukuro et al. 2017 for comparative morphological table of most deep water Neanthes spp.).

\section{Ecology}

Neanthes goodayi sp. nov. was found at depths ranging from 4000 to $4400 \mathrm{~m}$ living in crevices of polymetallic nodules (Fig. 8A-B), burrowing in xenophyophore foraminifera growing on nodules (Fig. 8C-E) or in mud balls on nodule surfaces (Fig. 8F-H). As in other nereidids, the strong eversible jaws, together with large eyes, indicate an active and predatory behaviour. While we were able to 

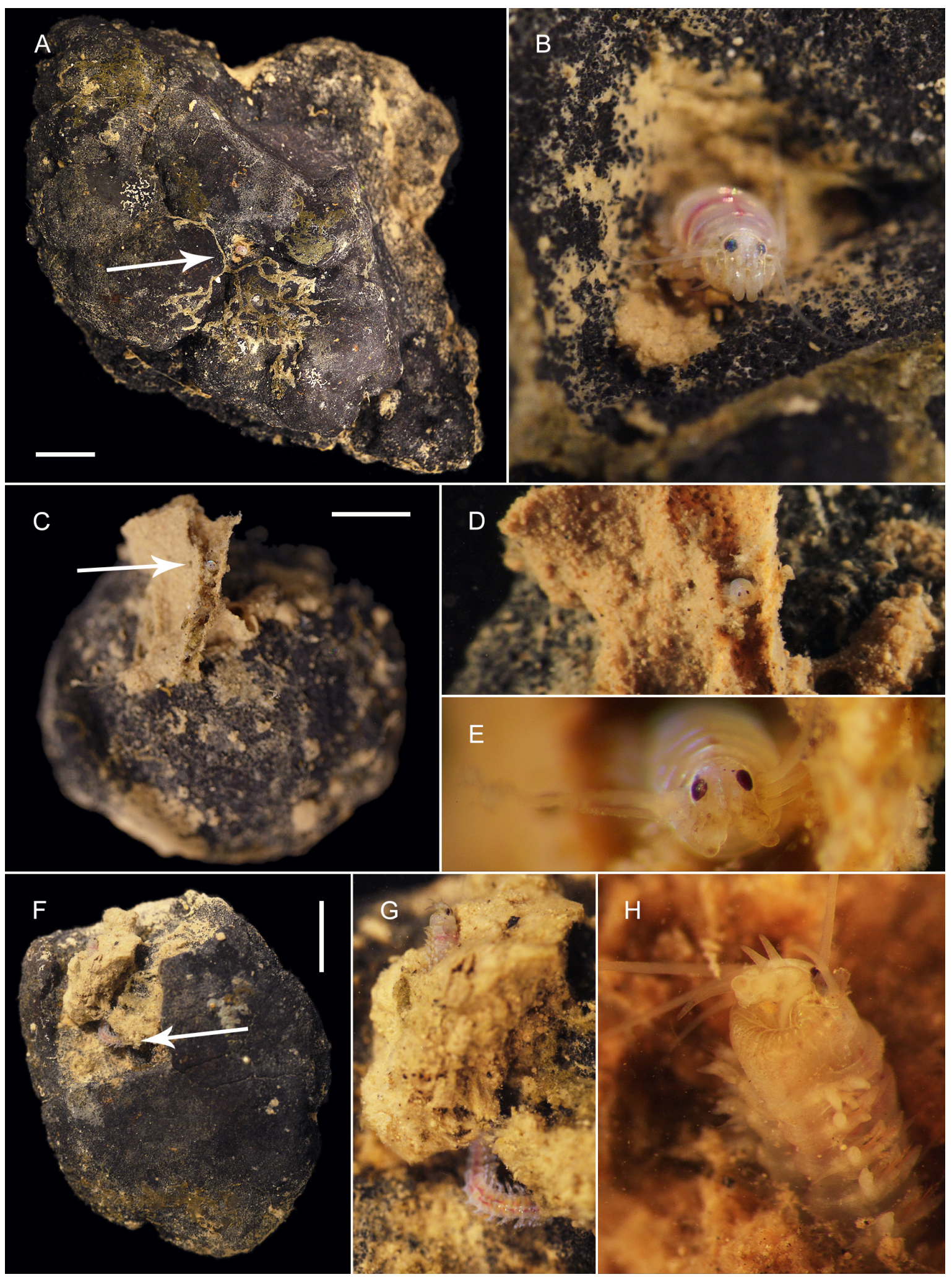

Fig. 8. Neanthes goodayi sp. nov., live specimens, in situ images. A. Paratype (NHM_2026), burrowing within nodule crevice. B. Detail of paratype (NHM_2026), in burrow. C. Paratype (NHM_512), burrowing within a foraminiferan growing on nodule. D-E. Detail of paratype (NHM_512), in burrow. F. Detail of paratype (NHM_1624), burrowing within a mudball encrusting the nodule surface. G-H. Details of paratype (NHM_1624), in burrow. Scale bars: $1 \mathrm{~cm}$. 
observe live, moving specimens kept at cold temperatures even after recovery from $4000 \mathrm{~m}$ water depth, behaviours such as predation were not observed. Polymetallic nodules are thought to contain a diverse meiofaunal community of nematodes, copepods and other small crustaceans; thus, it is possible that $N$. goodayi sp. nov. is a 'sit and wait' predator that is able to remain inside the nodules and detect prey passing overhead through extremely small variations in light (from local bioluminescence, detected by the large eyes) or other physio-chemical cues.

\section{Distribution}

Eastern Clarion Clipperton Fracture Zone, Central Eastern Pacific.

\section{Discussion}

It is perhaps remarkable that one of the more obvious and charismatic animals living on and inside the most investigated mineral resource on the deep seafloor has not been described until now. However, the $\mathrm{CCZ}$ region, despite a large number of expeditions and considerable sampling effort, has clearly never received appropriate taxonomic attention (Glover et al. 2018). Only in recent years has any effort been made to describe polychaete species, with 29 new species described in two recent papers (Bonafácio \& Menot 2018; Wiklund et al. 2019). Such descriptions are essential to future investigations of population connectivity and resilience, extinction risk modelling, ecosystem function, natural history, ecology and life history (Glover et al. 2018).

The more obvious macrofauna that live on polymetallic nodules are likely to be useful in the future for monitoring the impacts of seabed mining, if it were to start. In this regard, Neanthes goodayi can be added to this list of potential 'indicator taxa' alongside the recently described nodule-dwelling sponge, Plenaster craigi Lim \& Wiklund, 2017. Like P. craigi, $N$. goodayi sp. nov. is relatively easy to recognise during routine examination of nodules, and is sufficiently abundant to be counted in replicated samples. The smaller macrofauna dwelling in the sediments around the nodules is still extremely difficult to identify without using genetic methods and as such can only really be identified by specialists. The presence or absence of nodule-dwelling taxa such as $P$. craigi or $N$. goodayi sp. nov. may prove to be a useful measure of ecosystem health.

\section{Acknowledgements}

The UK Seabed Resources ABYSSLINE (ABYSSal baseLINE) environmental surveys were supported by a collaborative partnership between six non-profit global academic research institutes (University of Hawaii at Manoa, Natural History Museum, NORCE Norwegian Research Centre, National Oceanography Centre, Senckenberg Institute and Heriot-Watt University) and through an arrangement with UKSRL (UK Seabed Resources Ltd.). Additional support was provided by the Swedish research council FORMAS (TGD). We acknowledge Madeleine Brasier from the Natural History Museum team and Swee Cheng Lim from the National University of Singapore for support with sorting and sampling on board ship, and Chief Scientist Craig R. Smith for organizing the project and leading the sampling program. We also acknowledge the expert support from the Senckenberg Institute team in the deployment and recovery of successful Brenke epibenthic sledge samples. This study was made possible only by the dedicated help of the entire scientific party, the masters and crew of the RV Melville during the first cruise of the ABYSSLINE project in October 2013 and the masters and crew of the RV Thomas G. Thompson during the second ABYSSLINE cruise in February and March 2015. We sincerely thank reviewers for their effort in providing detailed comments and suggestions, which helped improve and clarify this manuscript. RD would like to thank and acknowledge support from the NERC INSPIRE DTP for the time needed to complete the manuscript. Thank you also to Emma Sherlock, Senior Curator of Annelida, Jackie Mackenzie-Dodds at the Molecular Collection Facility, and Harry Rousham and Robyn Fryer, Consultancy Group, all at the Natural History Museum. 


\section{References}

Amon D.J., Ziegler A.F., Dahlgren T.G., Glover A.G., Goineau A., Gooday A.J., Wiklund H. \& Smith C.R. 2016. Insights into the abundance and diversity of abyssal megafauna in a polymetallic-nodule region in the eastern Clarion-Clipperton Zone. Scientific Reports 6: e30492.

https://doi.org/10.1038/srep30492

Bakken T. 2006. Redescription of two species of Neanthes (Polychaeta: Nereididae) possessing a large notopodial prechaetal lobe. Scientia Marina 70 (Suppl. 3): 27-33.

https://doi.org/10.3989/scimar.2006.70s327

Bakken T. \& Wilson R.S. 2005. Phylogeny of nereidids (Polychaeta, Nereididae) with paragnaths. Zoologica Scripta 34 (5): 507-547. https://doi.org/10.1111/j.1463-6409.2005.00200.x

Bely A.E. \& Wray G.A. 2004. Molecular phylogeny of naidid worms (Annelida: Clitellata) based on cytochrome oxidase I. Molecular Phylogenetics and Evolution 30 (1): 50-63.

https://doi.org/10.1016/S1055-7903(03)00180-5

Bonifácio P. \& Menot L. 2018. New genera and species from the Equatorial Pacific provide phylogenetic insights into deep-sea Polynoidae (Annelida). Zoological Journal of the Linnean Society 185 (3): 555635. https://doi.org/10.1093/zoolinnean/zly063

Cohen B.L., Gawthrop A. \& Cavalier-Smith T. 1998. Molecular phylogeny of brachiopods and phoronids based on nuclear-encoded small subunit ribosomal RNA gene sequences. Philosophical Transactions of the Royal Society of London B 353 (1378): 2039-2061. https://doi.org/10.1098/rstb.1998.0351

Dahlgren T.G., Lundberg J., Pleijel F. \& Sundberg P. 2000. Morphological and molecular evidence of the phylogeny of nereidiform polychaetes (Annelida). Journal of Zoological Systematics and Evolutionary Research 38 (4): 249-253. https://doi.org/10.1046/j.1439-0469.2000.384150.x

Day J.H. 1963. The polychaete fauna of South Africa. Part 8: new species and records from grab samples and dredgings. Bulletin of the British Museum (Natural History), Series Zoology 10 (7): 381-445.

de León-González J.A. \& Solís-Weiss V. 2000. A review of the polychaete family Nereididae from western Mexico. Bulletin of Marine Science 67 (1): 549-569.

Droege G., Barker K., Seberg O., Coddington J., Benson E., Berendsohn W.G., Bunk B., Butler C., Cawsey E.M., Deck J., Döring M., Flemons P., Gemeinholzer B., Güntsch A., Hollowell T., Kelbert P., Kostadinov I., Kottmann R., Lawlor R.T., Lyal C., Mackenzie-Dodds J., Meyer C., Mulcahy D., Nussbeck S.Y., O'Tauma É., Orrell T., Petersen G., Robertson T., Söhngen C., Whitacre J., Wieczorek J., Yilmaz P., Zetzsche H., Zhang Y. \& Zhou X. 2016. The Global Genome Biodiversity Network (GGBN) Data Standard specification. Database 2016: 1-11. https://doi.org/10.1093/database/baw 125

Edgar R.C. 2004. MUSCLE: multiple sequence alignment with high accuracy and high throughput. Nucleic Acids Research 32 (5): 1792-1797. https://doi.org/10.1093/nar/gkh340

European Commission. 2020. Blue growth. Available from https://ec.europa.eu/maritimeaffairs/policy/blue_growth_en [accessed 17 Mar. 2020].

Fauchald K. 1972. Benthic polychaetous annelids from deep water off western Mexico and adjacent areas in the eastern Pacific Ocean. Allan Hancock Monographs in Marine Biology 7: 1-575.

Fauchald K. 1977. The polychaete worms. Definitions and keys to the orders, families and genera. Natural History Museum of Los Angeles County, Science Series 28: 1-188.

Fauchald K. \& Jumars P.A. 1979. The diet of worms: a study of polychaete feeding guilds. Oceanography and Marine Biology Annual Review 17: 193-284. 
Folmer O., Black M., Hoeh W., Lutz R. \& Vrijenhoek R. 1994. DNA primers for amplification of mitochondrial cytochrome c oxidase subunit I from diverse metazoan invertebrates. Molecular Marine Biology Biotechnology 3 (5): 294-299.

Glasby C.J., Wilson R.S. \& Bakken T. 2011. Redescription of the Indo-Pacific polychaete Neanthes pachychaeta (Fauvel, 1918) n. comb. (Annelida, Phyllodocida, Nereididae) and its synonyms. Zoosystema 33 (3): 361-375. https://doi.org/10.5252/z2011n3a5

Glover A.G., Dahlgren T.G., Wiklund H., Mohrbeck I. \& Smith C.R. 2016. An end-to-end DNA taxonomy methodology for benthic biodiversity survey in the Clarion-Clipperton Zone, Central Pacific abyss. Journal of Marine Science and Engineering 4 (1): e2. https://doi.org/10.3390/jmse4010002

Glover A.G., Wiklund H., Chen C. \& Dahlgren T.G. 2018. Point of view: managing a sustainable deepsea "blue economy" requires knowledge of what actually lives there. eLife 7: e41319.

https://doi.org/10.7554/eLife.41319

Gooday A.J., Holzmann M., Caulle C., Goineau A., Kamenskaya O., Weber A.A.T. \& Pawlowski J. 2017. Giant protists (xenophyophores, Foraminifera) are exceptionally diverse in parts of the abyssal eastern Pacific licensed for polymetallic nodule exploration. Biological Conservation 207: 106-116. https://doi.org/10.1016/j.biocon.2017.01.006

Hartman O. 1954. Australian Nereidae including descriptions of three new species and one genus, together with summaries of previous records and keys to species. Transactions of the Royal Society of South Australia 77: 1-41.

Hein J.R., Mizell K., Koschinsky A. \& Conrad T.A. 2013. Deep-ocean mineral deposits as a source of critical metals for high- and green-technology applications: comparison with land-based resources. Ore Geology Reviews 51: 1-14. https://doi.org/10.1016/j.oregeorev.2012.12.001

Hsueh P.W. 2019. Neanthes (Annelida: Nereididae) from Taiwanese waters, with description of seven new species and one new species record. Zootaxa 4554 (1): 173-198.

https://doi.org/10.11646/zootaxa.4554.1.5

Ibrahim N.F., Ibrahim Y.S. \& Sato M. 2019. New record of an estuarine polychaete, Neanthes glandicincta (Annelida, Nereididae) on the eastern coast of peninsular Malaysia. ZooKeys 831: 81-94.

https://doi.org/10.3897/zookeys.831.28588

ISA. 2018. International Seabed Authority - Exploration Areas. Available from

https://www.isa.org.jm/contractors/exploration-areas [accessed 12 Jul. 2021].

Jumars P.A., Dorgan K.M. \& Lindsay S.M. 2015. Diet of Worms emended: an update of polychaete feeding guilds. Annual Review of Marine Science 7 (1): 497-520.

https://doi.org/10.1146/annurev-marine-010814-020007

Katoh K. 2002. MAFFT: a novel method for rapid multiple sequence alignment based on fast Fourier transform. Nucleic Acids Research 30 (14): 3059-3066. https://doi.org/10.1093/nar/gkf436

Kearse M., Moir R., Wilson A., Stones-Havas S., Cheung M., Sturrock S., Buxton S., Cooper A., Markowitz S., Duran C. \& Thierer T. 2012. Geneious Basic: an integrated and extendable desktop software platform for the organization and analysis of sequence data. Bioinformatics 28 (12):16471649. https://doi.org/10.1093/bioinformatics/bts199

Kersken D., Janussen D. \& Martinez Arbizu P. 2019. Deep-sea glass sponges (Hexactinellida) from polymetallic nodule fields in the Clarion-Clipperton Fracture Zone (CCFZ), northeastern Pacific: Part II-Hexasterophora. Marine Biodiversity 49 (2): 947-987. https://doi.org/10.1007/s12526-018-0880-y 
Khlebovich V.V. 1996. Polychaetous Annelids, Volume III. Polychaetes of the family Nereididae of the Russian Seas and the adjacent waters. Fauna of Russia and Neighbouring Countries 140: 1-221.

Kinberg J.G.H. 1865. Annulata nova. Nereidum dispositio nova. Öfversigt af Kongeliga Vetenskapsakademiens Förhandlingar 22: 167-179.

Lim S-C., Wiklund H., Glover A.G., Dahlgren T.G. \& Tan K-S. 2017. A new genus and species of abyssal sponge commonly encrusting polymetallic nodules in the Clarion-Clipperton Zone, East Pacific Ocean. Systematics and Biodiversity 15 (6): 507-519. https://doi.org/10.1080/14772000.2017.1358218

Medlin L., Elwood H.J., Stickel S. \& Sogin M.L. 1988. The characterization of enzymatically amplified eukaryotic 16S-like rRNA-coding regions. Gene 71 (2): 491-499.

https://doi.org/10.1016/0378-1119(88)90066-2

Nygren A. \& Sundberg P. 2003. Phylogeny and evolution of reproductive modes in Autolytinae (Syllidae, Annelida). Molecular Phylogenetics and Evolution 29 (2): 235-249.

https://doi.org/10.1016/S1055-7903(03)00095-2

Palumbi S.R. 1996. Nucleic acids II: the polymerase chain reaction. Molecular Systematics 2 (1): 205247.

Posada D. 2008. jModelTest: Phylogenetic model averaging. Molecular Biology and Evolution 25: 1253-1256. https://doi.org/10.1093/molbev/msn083

Read G.B. 2007. Taxonomy of sympatric New Zealand species of Platynereis, with description of three new species additional to P. australis (Schmarda) (Annelida: Polychaeta: Nereididae). Zootaxa 1558 (1): 1-28. https://doi.org/10.11646/zootaxa.1558.1.1

Read G. \& Fauchald K. 2020a. World Polychaeta database. Nereididae Blainville, 1818. Available from http://www.marinespecies.org/aphia.php?p=taxdetails\&id=22496 [accessed 5 Feb. 2020].

Read G. \& Fauchald K. 2020b. World Polychaeta database. Neanthes Kinberg, 1865. Available from http://www.marinespecies.org/aphia.php?p=taxdetails\&id=129378 [accessed 5 Feb. 2020].

Ronquist F., Teslenko M., Van Der Mark P., Ayres D.L., Darling A., Höhna S., Larget B., Liu L., Suchard M.A. \& Huelsenbeck J.P. 2012. MrBayes 3.2: efficient Bayesian phylogenetic inference and model choice across a large model space. Systematic Biology 61 (3): 539-542.

Retrieved from https://doi.org/10.1093/sysbio/sys029

Sato M. 2013. Resurrection of the genus Nectoneanthes Imajima, 1972 (Nereididae: Polychaeta), with redescription of Nectoneanthes oxypoda (Marenzeller, 1879) and description of a new species, comparing them to Neanthes succinea (Leuckart, 1847). Journal of Natural History 47 (1-2): 1-50. https://doi.org/10.1080/00222933.2012.743609

Shimabukuro M., Santos C.S.G., Alfaro-Lucas J.M., Fujiwara Y. \& Sumida P.Y.G. 2017. A new eyeless species of Neanthes (Annelida: Nereididae) associated with a whale-fall community from the deep Southwest Atlantic Ocean. Deep Sea Research II 146: 27-34.

https://doi.org/10.1016/J.DSR2.2017.10.013

Sjölin E., Erséus C. \& Källersjö M. 2005. Phylogeny of Tubificidae (Annelida, Clitellata) based on mitochondrial and nuclear sequence data. Molecular Phylogenetics and Evolution 35 (2): 431-441. https://doi.org/10.1016/j.ympev.2004.12.018

Thiel H., Schriever G., Bussau C. \& Borowski C. 1993. Manganese nodule crevice fauna. Deep-Sea Research I 40 (2): 419-423. https://doi.org/10.1016/0967-0637(93)90012-R 
Villalobos-Guerrero T.F. 2019. Redescription of two overlooked species of the Perinereis nuntia complex and morphological delimitation of $P$. nuntia (Savigny in Lamarck, 1818) from the Red Sea (Annelida, Nereididae). Zoosystema 41 (1): 465-496. https://doi.org/10.5252/zoosystema2019v41a24

Villalobos-Guerrero T.F. \& Bakken T. 2018. Revision of the Alitta virens species complex (Annelida: Nereididae) from the North Pacific Ocean. Zootaxa 4483 (2): 1-257.

https://doi.org/10.11646/zootaxa.4483.2.1

Weigert A. \& Bleidorn C. 2016. Current status of annelid phylogeny. Organisms Diversity and Evolution 16 (2): 345-362. https://doi.org/10.1007/s13127-016-0265-7

Wieczorek J., Bloom D., Guralnick R., Blum S., Döring M., Giovanni R., Robertson T. \& Vieglais D. 2012. Darwin Core: An evolving community-developed biodiversity data standard. PLoS One 7 (1): e29715. https://doi.org/10.1371/journal.pone.0029715

Wiklund H., Neal L., Glover A.G., Drennan R., Rabone M. \& Dahlgren T.G. 2019. Abyssal fauna of polymetallic nodule exploration areas, eastern Clarion-Clipperton Zone, central Pacific Ocean: Annelida: Capitellidae, Opheliidae, Scalibregmatidae, and Travisiidae. ZooKeys 883: 1-82. https://doi.org/10.3897/zookeys.883.36193

Wilson R.S. 1984. Neanthes (Polychaeta: Nereididae) from Victoria with descriptions of two new species. Proceedings of the Royal Society of Victoria 96 (4): 209-226.

Wilson R.S. 1988. Synonymy of the genus Nectoneanthes Imajima, 1972, with Neanthes Kinberg, 1866 (Polychaeta: Nereididae). Proceedings of the Biological Society of Washington 101 (1): 4-10.

Wu P., Sun J. \& Yang T. 1985. Nereidae (Polychaetous Annelids) of the Chinese Coast. Springer-Verlag, Berlin, New York.

Manuscript received: 22 May 2020

Manuscript accepted: 8 June 2021

Published on: 27 July 2021

Topic editor: Rudy Jocqué

Desk editor: Danny Eibye-Jacobsen

Printed versions of all papers are also deposited in the libraries of the institutes that are members of the EJT consortium: Muséum national d'histoire naturelle, Paris, France; Meise Botanic Garden, Belgium; Royal Museum for Central Africa, Tervuren, Belgium; Royal Belgian Institute of Natural Sciences, Brussels, Belgium; Natural History Museum of Denmark, Copenhagen, Denmark; Naturalis Biodiversity Center, Leiden, the Netherlands; Museo Nacional de Ciencias Naturales-CSIC, Madrid, Spain; Real Jardín Botánico de Madrid CSIC, Spain; Zoological Research Museum Alexander Koenig, Bonn, Germany; National Museum, Prague, Czech Republic.

Supplementary file 1. DarwinCore Archive (DWcA) of all specimen occurrence (and associated preparation) data. https://doi.org/10.5852/ejt.2021.760.1447.4755 